\title{
Hydromechanical Coupling and Pit Slope Movements
}

\author{
T.D. Sullivan Pells Sullivan Meynink Pty Ltd and The University of New South Wales, Australia
}

\begin{abstract}
This paper presents a review of the general state of the art in the understanding of the interaction between water and pit slope deformations. The topics covered include; the theory of hydromechanical coupling, the interaction between stress and pore fluid responses for rock structure and rock masses and the impacts of these on open pit slopes.
\end{abstract}

The hydrogeological cycle for an open pit is explained and the importance of transient and partial pore pressures are highlighted. The paper includes an explanation of pit slope deformations and failure movements. A new system for classifying pit slope movements is presented together with critical movement thresholds.

The various methods for slope depressurisation are addressed and the accuracy and methodology for assessing pore pressure input to slope design at all slope scales are reviewed.

Examples of hydromechanical coupling, pore pressure responses in structure and rock masses, statistics on the interaction between rainfall and pit slope failures and the role of undrained loading for the liquefaction potential for pit slopes are presented.

It is concluded that the effective management of pit slopes is only possible by the integration of pit slope deformations and movements with the hydraulic properties and pore pressure responses of the rock mass.

\section{Introduction}

This paper sets out to present a review of the general state of the art in the understanding of the interaction between water and pit slopes. The section of the earth's crust through which most mine slopes are excavated is porous and hence these slopes and the groundwaters contained therein, including water introduced from rainfall runoff, are intimately linked through their mechanical effects on each other (Neuzil, 2003). This process is termed "hydromechanical" coupling. Hydromechanical coupling is the physical interaction between hydraulic and mechanical processes.

The emphasis in this paper is on understanding the impacts of mining on natural hydrological processes and the hydrogeology of rock masses. Particular elements addressed here are the integration of slope deformations and pit wall movements on the hydrological performance. These elements are integrated through the principles of rock mechanics, soil mechanics, hydrogeology and geology.

The topic is very broad and it is not possible to cover all the detail, thus this paper focuses on the concepts and the practical elements. This paper deals mainly with open pit slopes although the general concepts relate at least in part to all excavated rock slopes.

The understanding of the physical interactions between fluids and the medium that contains them was developed a long time ago to explain separate physical responses of our natural world. The aim of this paper is to collate and bring together into a common body of understanding the theories and work in the fields of geotechnical engineering (Terzaghi, 1923), aquifer response in groundwater hydrology and Biot's equations for isotropic linear elastic porous media; and then to place these ideas and understanding into the context of the responses and behaviours we observe with pit slopes.

The large scale performance of pit slopes over the life of the mine is potentially influenced by many "environmental" factors. Understanding these influences leads to the better recognition of risks and the better management of those risks. 


\section{Hydromechanical coupling}

\subsection{Introduction}

The theory of hydromechanical coupling in natural geological processes and in fractured rocks is covered extensively by Neuzil (2003) and Rutqvist and Stephansson (2003).

However despite the fundamental importance of this process to pit slope performance, most engineers pay scant attention to the phenomena in their designs and predictions of future performance. In addition many hydrogeologists tend to think in terms of fixed hydraulic parameters, failing to understand the effects of pit slope deformations and the impacts of those on hydraulic properties and transient groundwater effects.

Pore water controls the deformation of porous media and in turn the deformation of porous media affects pore fluid pressures and flows, mainly by altering hydraulic conductivity and storage. This is a two way process and is a fundamental aspect of the dynamic interaction that affects the deformation of pit slopes and the groundwaters contained within them.

\subsection{Theory of hydromechanical coupling}

The most simple theoretical example of hydromechanical coupling in soils is the theory of effective stress (Terzaghi, 1923). This theory was developed initially to explain the consolidation of soils over time and the dissipation of pore fluid pressures by flow. The vertical effective stress $\left(\sigma^{\prime}\right)$ is equal to total stress, or the applied load $\left(\sigma_{n}\right)$, minus the pressure in the pores or joints $(\mu)$; where:

$$
\sigma^{\prime}=\sigma_{n}-\mu
$$

This is the fundamental equation for describing hydromechanical coupling. The concept of effective stress lies at the core of the understanding of hydromechanical coupling.

In describing the consolidation response the two key parameters are Specific Storage $\left(S_{s}\right)$, which is the volume of water released per unit drop in head, and hydraulic conductivity $(K)$. In soil mechanics it is usual to describe consolidation in terms of the Coefficient of Consolidation, $c_{v}$. In groundwater hydrology, hydraulic conductivity and storage are also key parameters, although the terms normally used are Transmissivity $(T)$ and Storage Coefficient $(S)$. Transmissivity is equal to the hydraulic conductivity $(K)$ times the saturated thickness. However overall these two different approaches to describing the response of a material to changes in water are the same, where:

$$
c_{v}=\frac{K}{S_{s}}=\frac{T}{S}
$$

These ideas were built on with the development of the "General Theory of Three Dimensional Consolidation" or poroelastic theory (Biot, 1941) and its subsequent theories. However these theories proved to have limited application in geology because of the complexity of most geological terrains.

The main problem with poroelasticity is the assumption of very small linear elastic strains, which is nonrepresentative of most geological materials, particularly the deformations of pit slopes. Many pit slopes undergo large unrecoverable deformations, which are a complex function of the three dimensional stress state, time, geotechnical properties of the rock mass, the structural geology, groundwater pressures and excavation practices.

\subsection{Hydromechanical coupling and rock behaviour}

Theory and experience show that pit slopes display a range of behaviours including; elastic, non-linear elastic, elastoplastic and viscoelastic (Sullivan, 1993). This is illustrated in Figures 1 and 2, which show a number of different types of plastic yielding and nonlinear elastic, viscoelastic (creep) and elastoplastic behaviour for rocks. In the transition from intact rock to rock masses, from continuum to discontinuum mechanics, these behavioural patterns become complicated due to the introduction of structure and induced fracturing due to deformation. 


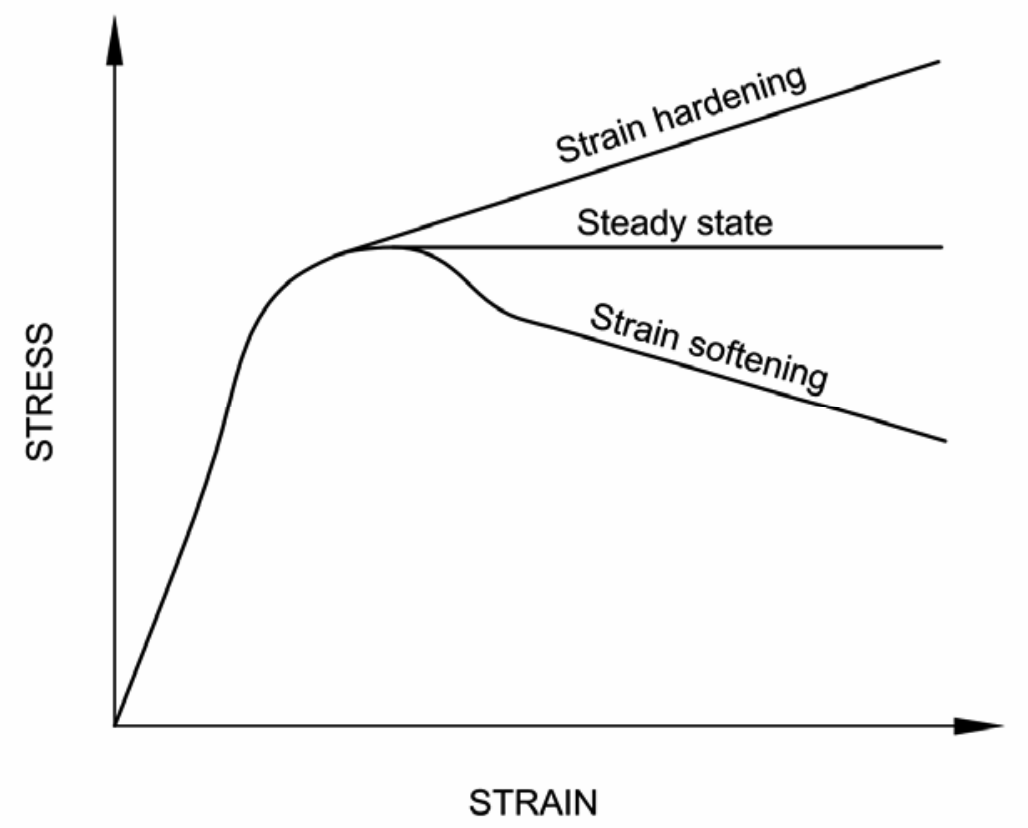

Figure 1 Stress versus strain showing the types of plastic yielding observed in rocks

Mohr diagrams are useful for considering the relationship between stresses, pore pressures and slip on geological structures or failure of the rock mass. Figure 3 shows this relationship. Shear failure of the rock or slip along pre-existing discontinuity, can occur due to either an increase in the major principal stress $\left(\sigma_{1}\right)$ or a decrease in the minimum principal stress $\left(\sigma_{3}\right)$, which in open pit mining could equate to either continued deepening of the pit or an increase in the pore fluid pressure, respectively.

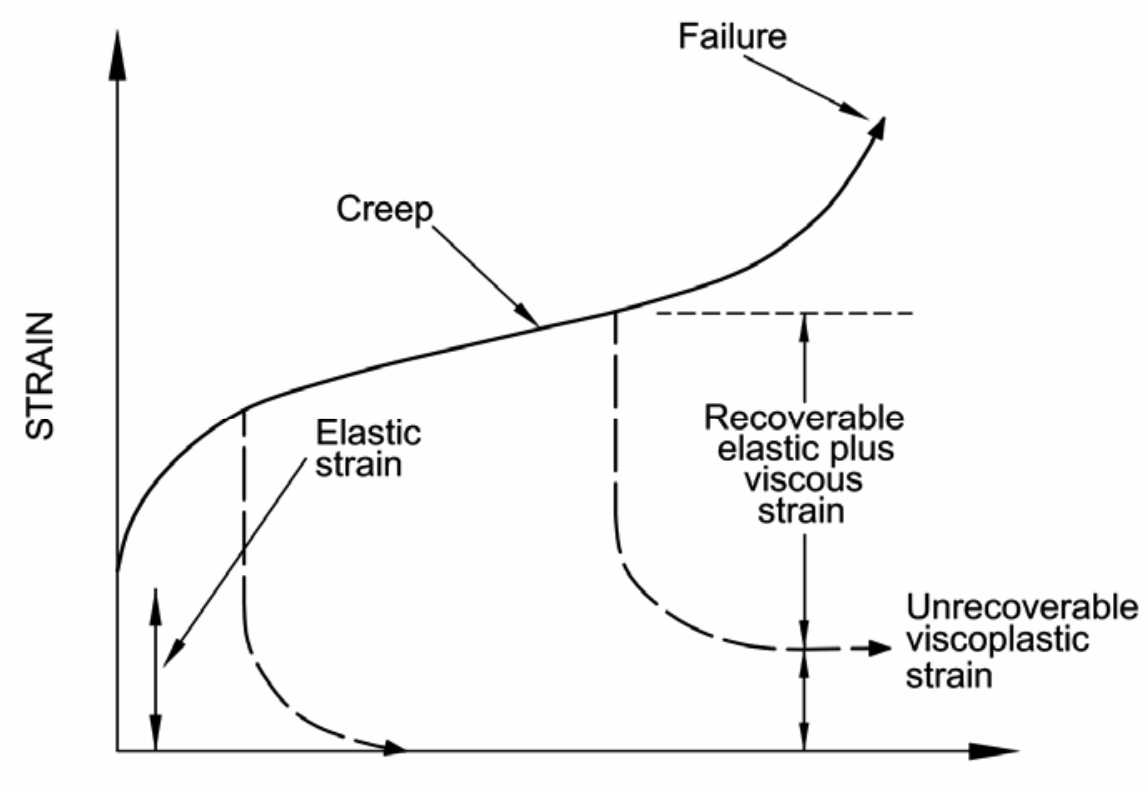

TIME

Figure 2 Strain versus time with typical viscoelastic behaviour of rock, including; non-linear elastic, viscoelastic and elastoplastic behaviour 


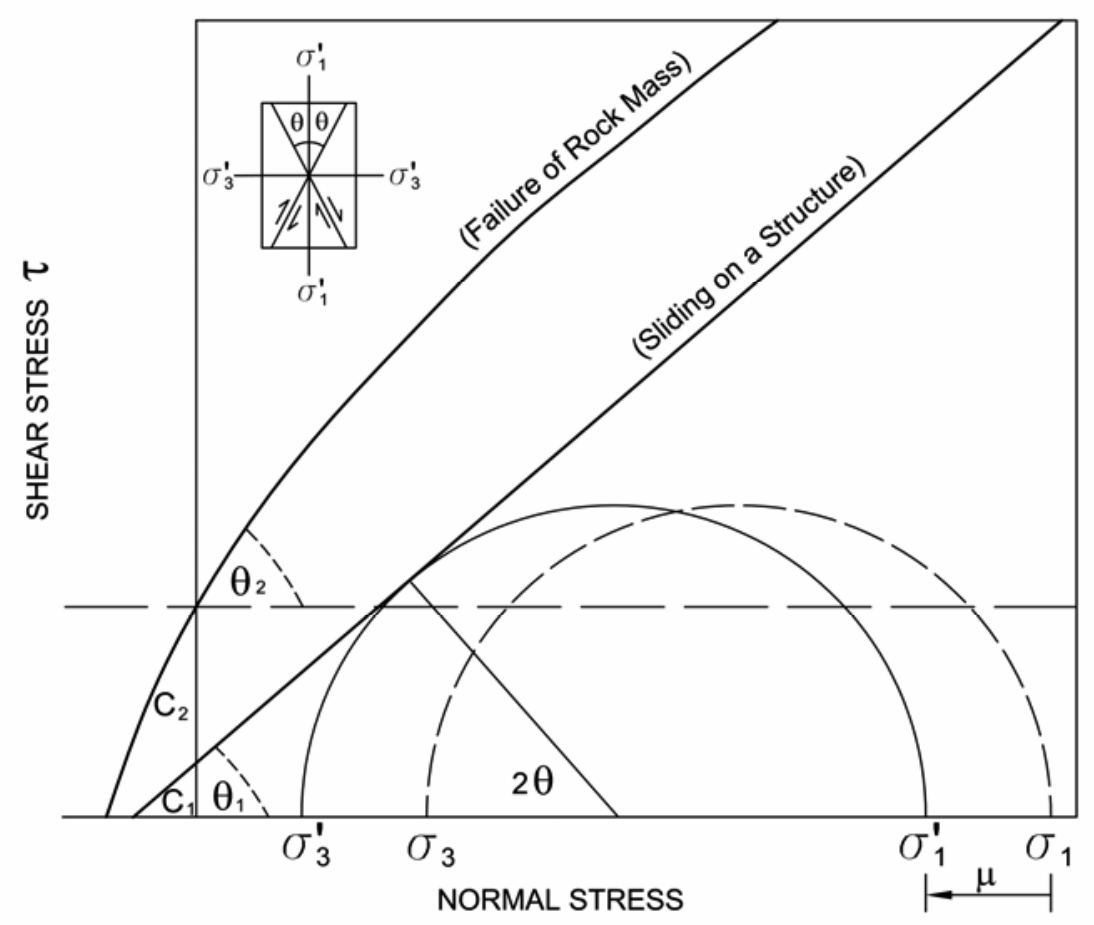

Figure 3 Mohr circle diagram showing the failure envelopes for shear failure of rock mass and sliding on a structure; where $\sigma_{1}$ and $\sigma_{3}$ are total stresses and $\sigma_{1}{ }^{\prime} \sigma_{3}{ }^{\prime}$ are effective stresses. Pore fluid pressure $(\mu)$ translates the Mohr circle to the left causing sliding on the structure. The same mechanism would apply to failure of the rock mass

Typically in open pits shear displacement occurs on structures. This can be continuous over time or more typically for large failures, cyclic with "stick-slip" behaviour often related to periodic rainfall and groundwater pressure build-up.

\subsection{Types of hydromechanical coupling}

Rutqvist and Stephansson (2003) and Wang (2000) recognize two types of hydromechanical coupling; direct and indirect. Direct coupling occurs through deformation and pore fluid interactions. Indirect coupling is where changes in the mechanical or hydraulic processes affect each other through changes in mechanical and hydraulic properties. The four types of hydromechanical coupling are illustrated in Figure 4.

Direct coupling entails:

- Solid to fluid coupling, when a change in the stress causes a change in the pore fluid pressure (Type 1).

- Fluid to solid coupling, when a change in the pore fluid pressure causes a change in the rock mass volume (Type 2).

These two cases relate predominantly to changes in the pore volume, which will also result in a change to the mechanical properties.

Indirect coupling entails:

- Solid to fluid coupling, where a change in the stress causes a change in the hydraulic properties of the rock mass (Type 3).

- Fluid to solid coupling, where a change in the pore fluid pressure causes a change in the mechanical properties of the rock mass (Type 4). 
DIRECT

TYPE 1
INDIRECT

TYPE 3

ACTION

RESULT

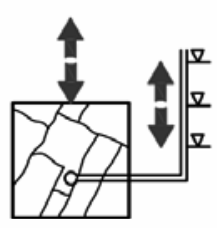

TYPE 2

TYPE 4
ACTION

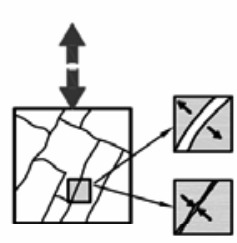
$\underset{(+o r-)}{\Delta \sigma}$
RESULT

\section{$\triangle K \& S$}

Hydraulic

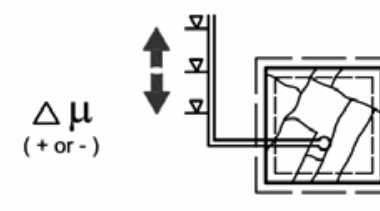

$\underset{(+o r-)}{\triangle \mathrm{V}}$

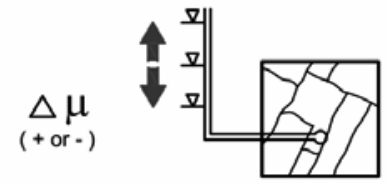

$\triangle E, k_{s}, k_{n}$

Mechanical

$c^{\prime}, \emptyset \quad$ Properties

Figure 4 Diagrammatic representation of direct and indirect hydromechanical coupling. Where $\Delta \sigma$ is a change in stress, $\Delta \mu$ is a change in pore pressure, $\Delta \mathrm{V}$ is a change in volume, $\mathrm{K}$ is hydraulic conductivity, $S$ is storage, $E$ is modulus of elasticity, $k_{s}$ is shear stiffness, $k_{n}$ is normal stiffness, $c$ is cohesion and $\Phi$ is angle of friction

The deformation of pit slopes, which are largely inelastic with creep and slip on structures, cause irreversible changes in the rock mass and hydraulic properties of the mass and are largely indirect coupling (Types 3 and 4). However liquefaction type failures are an example of initial direct hydromechanical coupling (Type 1) then leading to indirect hydromechanical coupling (Type 4).

\subsection{Rock masses}

In saturated rock masses deformation can occur as a result of a change in the stresses or a change in the pore fluid pressures. Figure 5 is a schematic representation of a heterogeneous rock mass forming a pit slope. This figure illustrates deformations, both shear and normal, for rock discontinuities and the rock mass. The rock mass is saturated and hence it is evident that any deformations in the rock mass will result in a change in the pore fluid pressure and a change in both the hydraulic conductivity and storage. Figure 6 shows the deformations which are illustrated in Figure 5. The dilation of rock structures; increased aperture, hydraulic conductivity, connectivity and storage due to the deformations are evident on the pit face.

\section{Pit slopes and hydromechanical coupling}

There is an under appreciation that many if not most of the geotechnical processes of pit slope deformation and behaviour can only be fully understood by considering the coupling between groundwater and deformation. These mechanical interactions underlie and provide a unifying view of many geotechnical and hydrogeological phenomena and this is still not widely appreciated.

In natural geological and geomorphological environments groundwater flow tends to be "quasi stable" and adjusted to the long term stress regime. But what happens when major disturbances to this regime occur as is the case with open pit mining? The natural trend is for the system to try and return to equilibrium unless the disturbance continues. Because mining continues we are in nearly all cases dealing with transient flow conditions. Deformation as a result of unloading (excavation) or loading leads to changes in the stresses resulting in changes in the pore fluid pressure resulting in transient flows, Figures 4 and 5. 


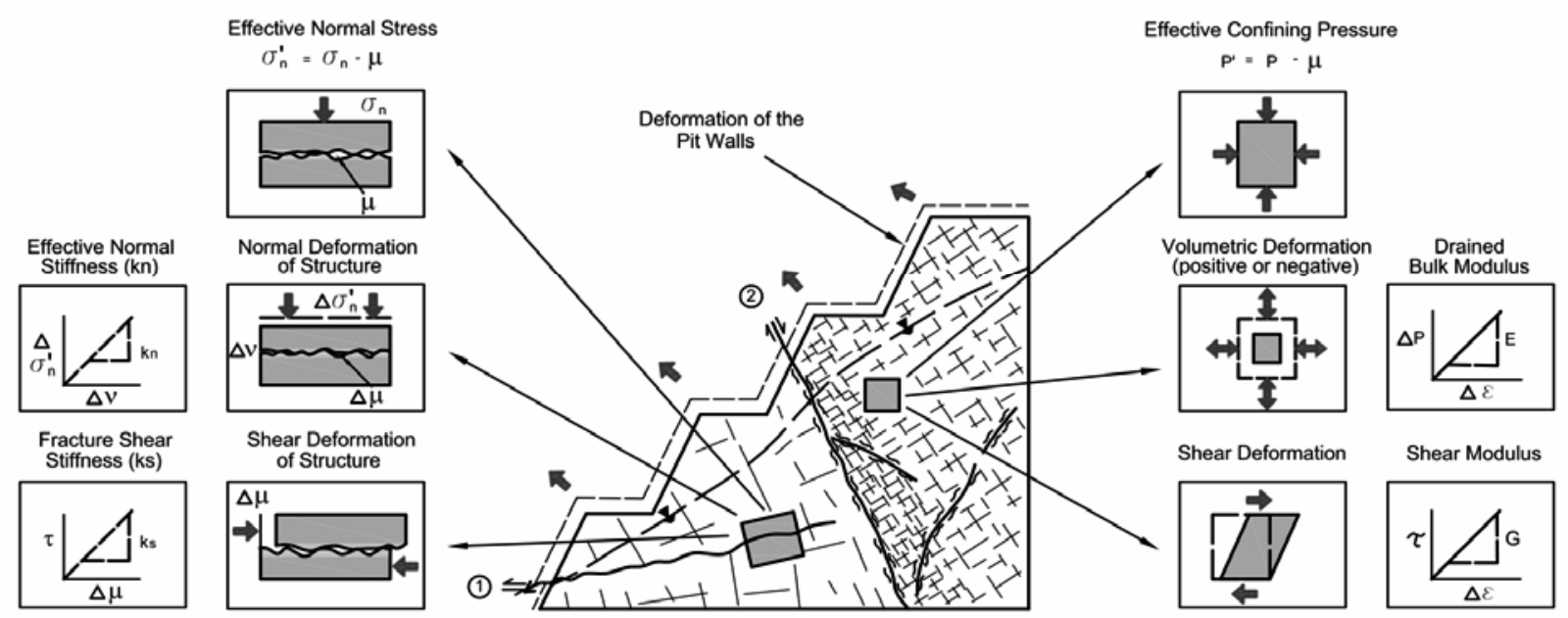

Figure 5 Schematic representations of hydromechanical coupling processes in a rock mass. Where $\sigma_{\mathrm{n}}{ }^{\prime}$ is effective normal stress, $\nu$ is normal displacement, $\tau$ is shear stress, $\mu$ is shear displacement, $\varepsilon$ is strain, $E$ is modulus and $G$ is shear modulus (after Rutqvist and Stephansson 2003). The deformations shown at points 1 and 2 in this figure are illustrated in Figure 6

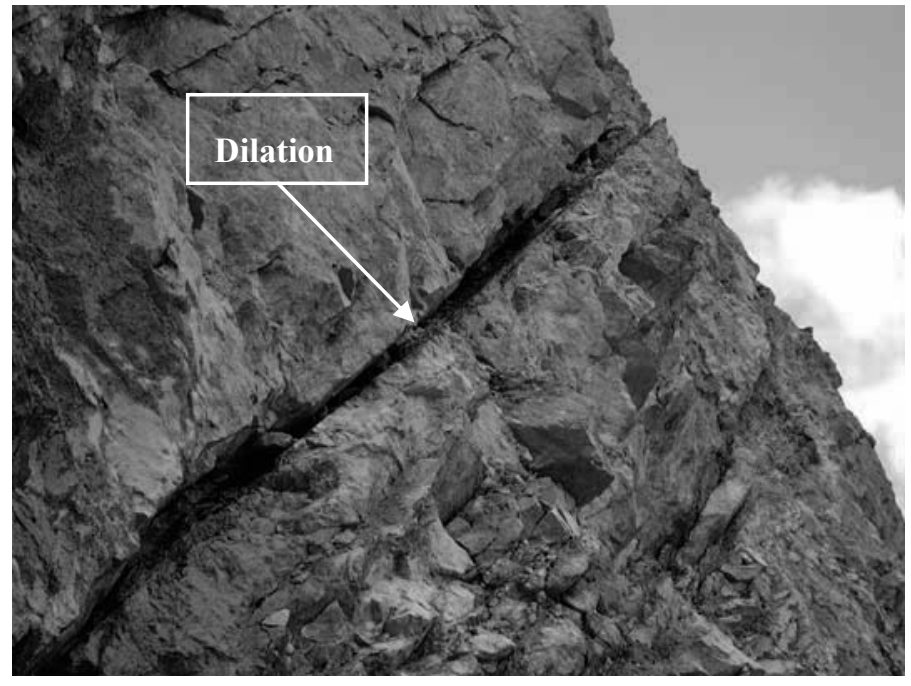

a)

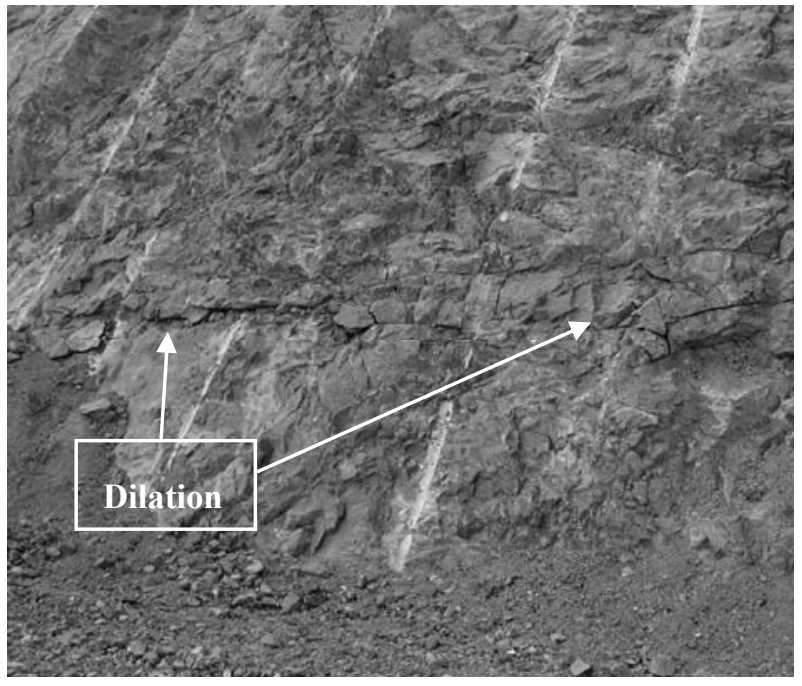

b)

Figure 6 Dilation, increased hydraulic conductivity and connectivity of structures in a pit wall, where a) is dilation due to shear on an undulating irregular subhorizontal structure and b) is dilation due to toppling (block rotation); refer to points 1 and 2 in Figure 5 respectively

Most natural slopes, with the exception of some geological settings, are quite old, have generally reached equilibrium with their environment and usually are quite stable. Similarly most slopes excavated in a civil engineering context are designed to be stable under the prevailing environmental conditions, with the stability generally evaluated for a range of conditions including large rainfall responses, leading in part to high factors of safety and or very low probabilities of failure. 
It is important to the understanding of the concepts presented in this paper that open pit slopes need to be differentiated from natural slopes firstly because in most cases the physical processes are probably changing at least in degree over the period of the mine life and secondly because these recently excavated slopes will be subject to "new" environmental influences from water effects.

Pit excavation reduces the lateral and vertical stress resulting in strains that generally increase porosity, aperture and connectivity between fractures. This is illustrated in Figures 4 and 5. Furthermore, over the mine life other deformations occur, such as creep or movement on structures, Figures 1 and 2. This creep or slip alters the nearby stress field and fluid pressures instantaneously. If stick-slip movement has occurred with no ongoing creep, then during the "stick" period, transient flows occur, which gradually dissipates the pore fluid pressure changes.

Furthermore many mine slopes are performance based designs. Implicit or explicit in this design process is an acceptance of some instability or a certain percentage of failure (Sullivan, 1994 and 2006). In addition in certain instances deformation based slope designs have been utilized. Hence the slopes are designed specifically in the knowledge that significant deformations will occur and that some failure is inherent in the design process. What are the implications of this on hydromechanical coupling and ultimately on slope performance?

Implications include:

- Because of the deformation and pit wall movements, there is an increased sensitivity to "environmental factors" such as rainfall-runoff.

- These "environmental factors" are often a new condition for the pit slope.

- This means there is usually a limited experience base to accurately predict the hydrogeological response and slope performance.

- Because the pit slope height is usually increasing throughout the mine life, the sensitivity of the slope to these "environmental factors" is also likely to change (usually increasing) over the mine life.

- It is very easy for the overall mine stability to change from an acceptable to an unacceptable level because of the impact of these external factors. This is particularly the case with a performance based slope designs.

- Hence it is relatively easy for the initial pit slope design assumptions to become invalid over time.

\section{$4 \quad$ Hydrological cycle for an open pit}

\subsection{Hydrological cycle}

In an open pit there are three main components of water; infiltration, surface flow and groundwater. These components form a complex interdependent system that is constantly changing throughout the mine life. This system is called the hydrological cycle. Figure 7 shows a schematic representation of the hydrological cycle for a typical open pit.

The principal elements to be considered in a hydrological cycle for an open pit are:

- Water sources:

○ Precipitation.

○ Runoff.

○ Groundwater inflow.

- Catchments, which are often different for ground and surface waters:

○ Surface water catchment.

○ Groundwater catchment. 
- Water extraction systems, including:
○ Sumps.
○ Wells.
○ Horizontal drains.
○ Drainage adits.

- Temporary or permanent water storages:
○ Sumps.
○ Ponds.

- Groundwater levels and pressures:

○ Groundwater table.

○ Interflow.

○ Transient pore pressures.

- Partial pore pressures below the groundwater table or level of saturation.

- Hydraulically significant changes to the rock mass surrounding the open pit as a result of mining, including:

- Fractured rock aquifer adjacent and sub-parallel to the pit walls.

- A movement zone surrounding the open pit with changed hydraulic conductivity and storage.

A component of the rainfall (precipitation) that falls inside the open pit surface catchment becomes runoff (Figure 7). The runoff is a function of precipitation intensity, precipitation duration, hydraulic conductivity of the ground surface, pit surface geometry and depth to the groundwater table. Surface runoff equals precipitation minus infiltration, surface retention and evaporation. Runoff is collected in swamps and temporary storages. A part of the water that infiltrates can be caught in the near surface zone, occurs as interflow and can report again as surface runoff, Figure 7. This usually occurs because of the presence of impervious layers or highly permeable structures, which in an open pit are geological structures or layers.

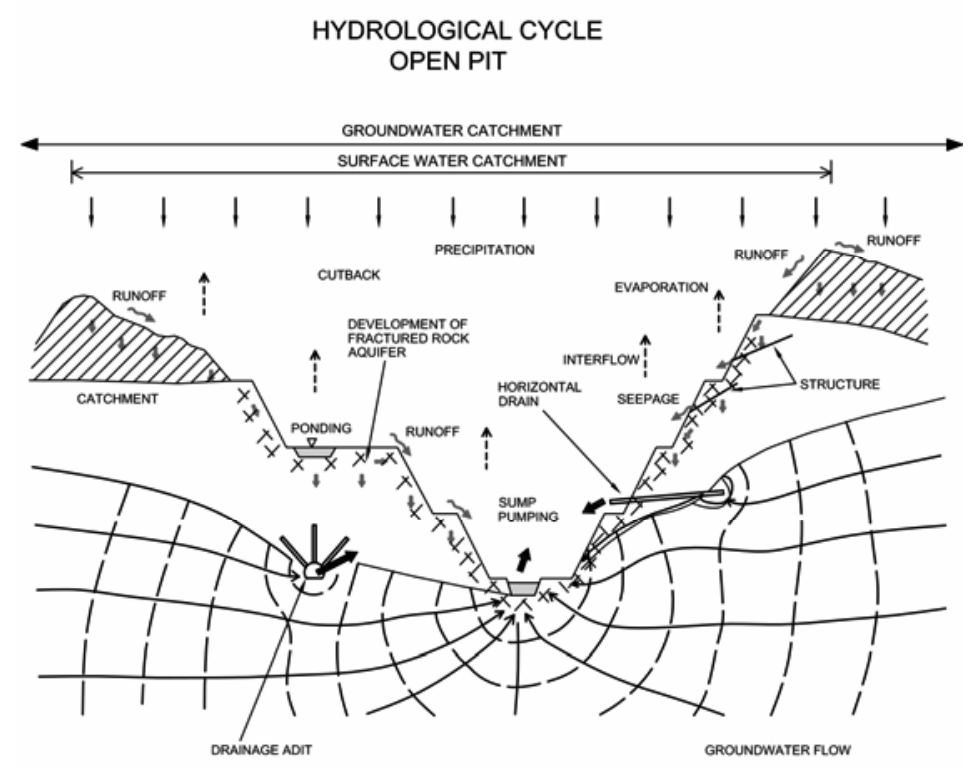

Figure 7 Hydrological cycle for a typical open pit 
In open pit mining there are also two other factors to account for that are not part of the natural environment; firstly the disturbance due to blasting for excavation, which results in a "fractured rock aquifer" parallel to the pit walls and secondly because of the deformations that occur due to unloading there is a change in hydraulic conductivity and storage usually increased, in a zone surrounding the open pit, but including the blasting induced fractured zone, Figure 7.

\subsection{Groundwater table, transient and partial pore pressures}

Given the scale of many pit slopes, which are now approaching and exceeding $1000 \mathrm{~m}$ depth, and the depth of dewatering and depressurisation required to maintain stability the roles of transient and partial pore pressures in the upper levels of many pit slopes are becoming increasingly important.

The simplest understanding of groundwater is associated with concepts of a groundwater table, with unsaturated conditions above and saturated conditions below it. However there is also the capillary fringe, which exists above the water table and while significant in soils can probably be ignored in most open pit situations. The groundwater table is best thought of as the surface where the pore pressure is atmospheric and this is the level at which water stands in a shallow open piezometers that just penetrates deep enough to encounter standing water.

Figure 8 illustrates some of these concepts with respect to a rock mass where there is a general downwards hydraulic gradient. Below the groundwater table, the rock mass is saturated, the moisture content is constant, the hydraulic conductivity is constant but the pore pressures may be equal to or less than hydrostatic ( $\mathrm{P}=$ $1 / 2 \gamma_{\mathrm{w}} \mathrm{h}^{2}$ ). Above the water table the pore pressures are negative.

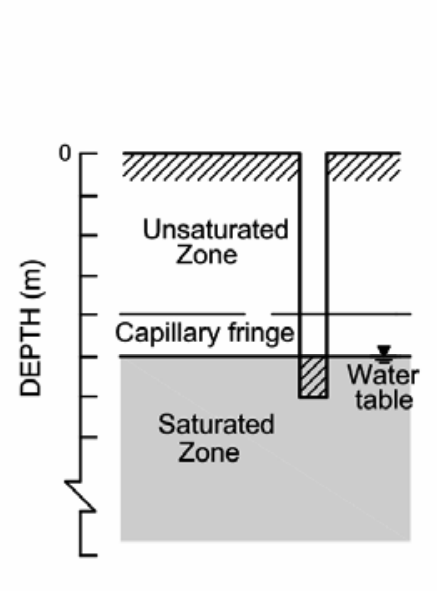

(a)

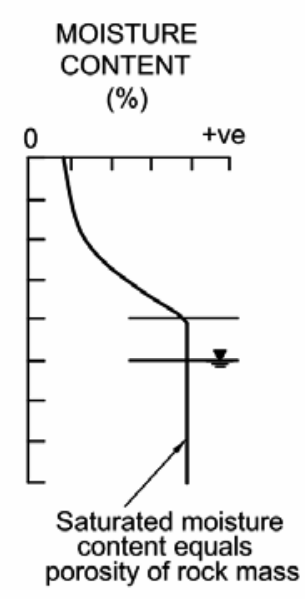

(b)

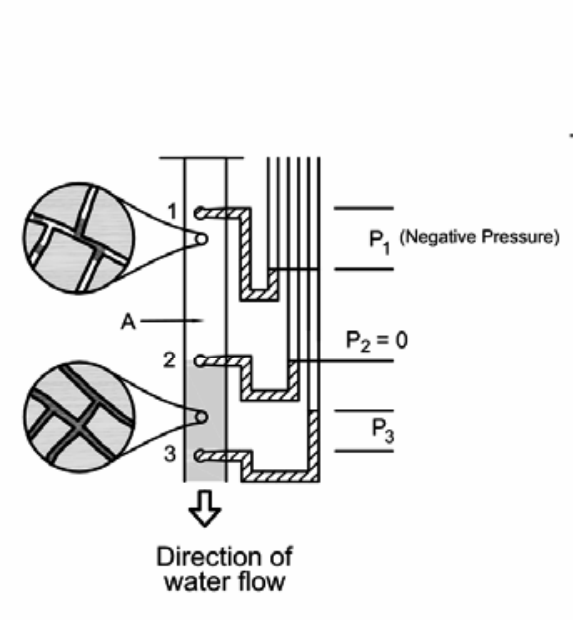

(c)

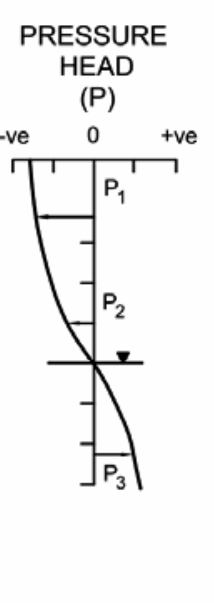

(d)

Figure 8 Groundwater conditions showing the relationship between the groundwater table, moisture content, degree of saturation and pore pressure (after Freeze and Cherry, 1979)

Infiltration at the groundsurface occurs due to rainfall. The rate of infiltration is termed the infiltration capacity and if during a rainfall event the rate of rainfall exceeds the infiltration capacity then ponding and runoff occur. Although it is not a constant, until saturation occurs, the infiltration rate is ultimately equal to the saturated hydraulic conductivity of the rock mass. Heavy precipitation leads to saturation at the ground surface. This results in transient saturated zones that build up and dissipate over time under the influence of infiltration. As illustrated in Figure 9 this condition results in transient water pressures in the zone above the deeper groundwater table. This is a temporary situation and the lower boundary of this upper saturated zone can be thought of as an inverted water table, Figure 9(b). 
Figure 9 illustrates the relationship between hydrostatic conditions below the groundwater table with full hydraulic pressure $\left(\mathrm{P}=1 / 2 \gamma_{\mathrm{w}} \mathrm{h}^{2}\right)$ and the partial pore pressure $\left(\mathrm{P}<1 / 2 \gamma_{\mathrm{w}} \mathrm{h}^{2}\right)$ due to underdrainage or pit wall depressurisation measures.

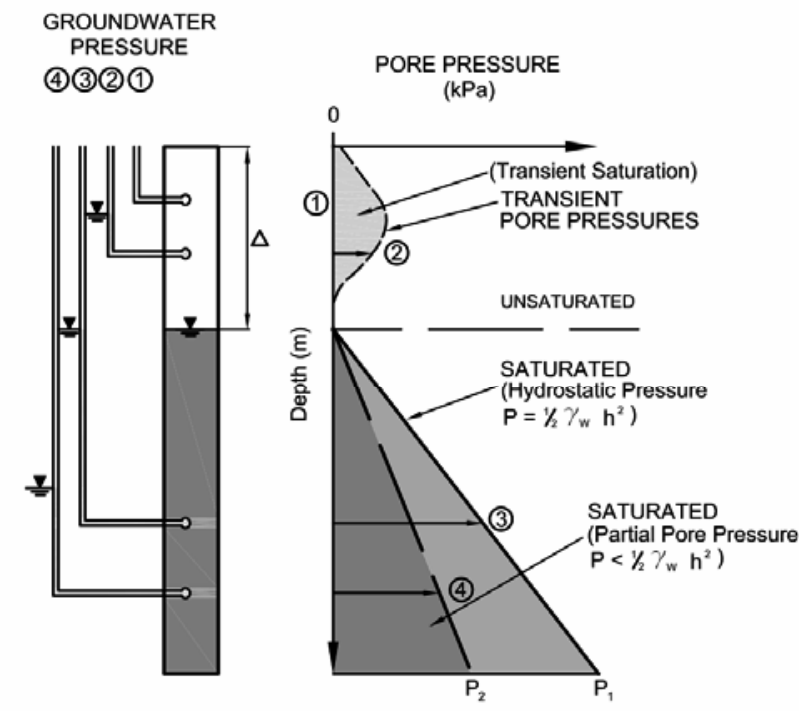

(a)

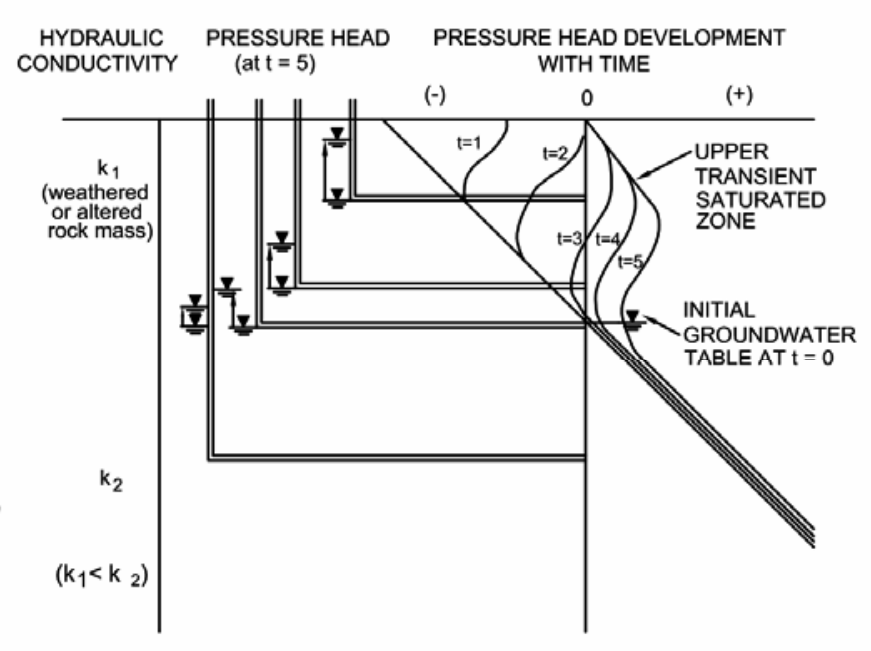

(b)

Figure 9 Illustration of the relationships between groundwater table, pore pressures and transient groundwater conditions. Figure 9(a), the relationship between unsaturated conditions (1), transient pore pressures above the groundwater table (2), hydrostatic groundwater pressures below the groundwater table (3) and partial pore pressures in the saturated zone below the groundwater table (4). Figure 9(b), the change in pressure head with time above the groundwater table due to infiltration at the ground surface. This is illustrated for a case with a lower permeability near surface zone

\section{Hydrogeological characteristics of rock masses}

\subsection{Hydraulic conductivity and depth}

Rock masses are clearly very heterogeneous (see Figure 5), and therefore both the mechanical properties and the hydraulic properties are also very heterogeneous. Various studies have shown that typically a rock mass contains a small number of highly transmissive fracture networks within a much larger network of lower permeability structures (Rutqvist and Stephansson, 2003).

Figure 10 shows the range of hydraulic conductivities measured with depth at a nuclear waste storage facility in granite (After Rutqvist and Stephansson 2003). The hydraulic conductivities cover a range of five orders of magnitude. Also evident is the effect of depth, or stress, which is most pronounced in the upper 200 to $300 \mathrm{~m}$. There are various explanations for this including, near surface stress relief, rebound after glaciation and or erosion; and the nonlinear stress dependency of fracture apertures, Figure 12. The upper bound permeabilities which occur even at considerable depth are high conductivity structures with open channels formed either by rock bridges or mineral cementation or fault zones with substantial shear dislocations. These high conductivity structures are not likely to be stress dependent. However tests have shown that the apertures of normal fractures are quite stress dependent and tests at one location showed a factor of three in hydraulic conductivity between fractures in a highly anisotropic stress field. Hence below a certain depth it is likely that most fractures are closed to their residual hydraulic conductivity, Figure 12. 


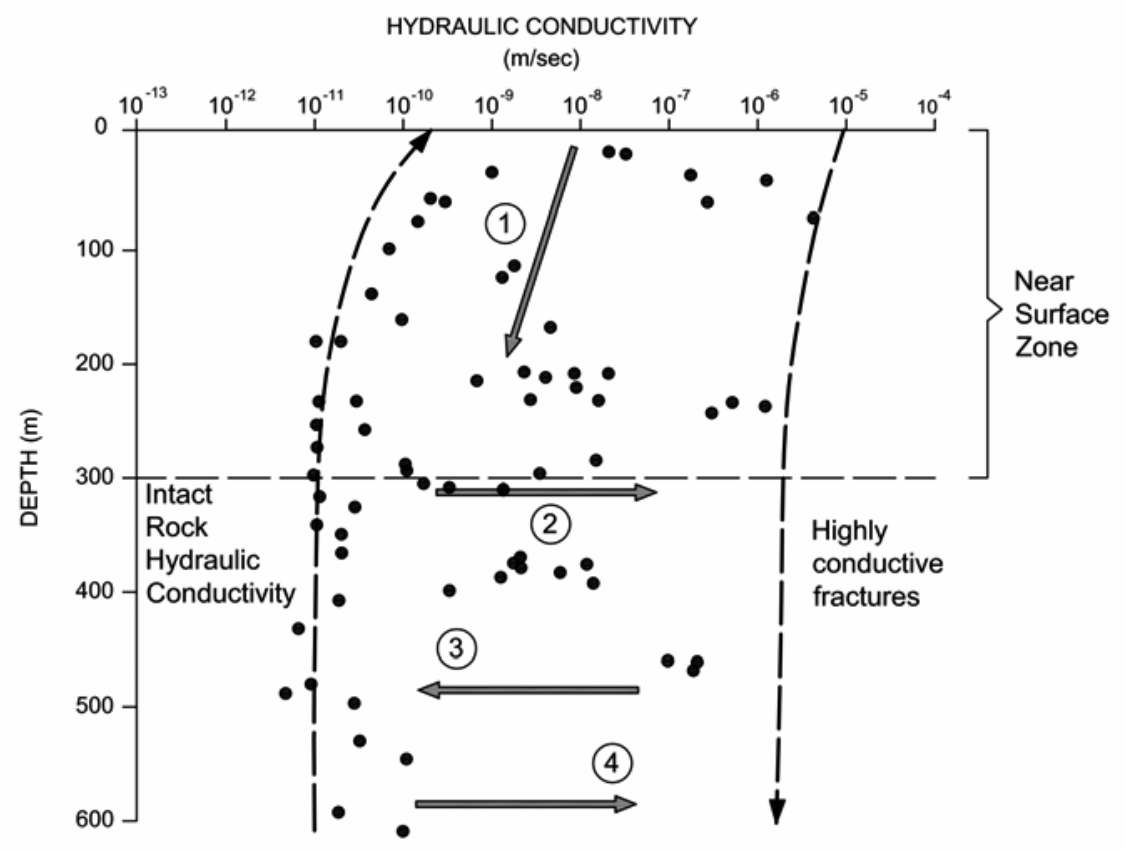

Figure 10 Plot of Hydraulic Conductivity with depth, where there is a reduction in hydraulic conductivity in the near surface zone due to increasing stress (1), shear displacement generally tend to increase hydraulic conductivity (2) and precipitation or dissolutions of minerals at depth can increase or decrease hydraulic conductivity (3 and 4) (after Rutqvist and Stephansson, 2003)

Fractures with a small initial hydraulic conductivity are likely to be more sensitive to changes in normal stress $\left(\sigma_{\mathrm{n}}\right)$ than fractures with a large initial hydraulic conductivity. Similarly, fractures with a large initial hydraulic conductivity are more likely to be longer and hence tend to have less dilation under shear than smaller fractures, thus they will be less sensitive to changes in hydraulic conductivity due to shearing. This is opposite to the effect with fractures with a small initial hydraulic conductivity, Figure 12.

The possible changes to hydraulic conductivity due to stress changes are indicated in Figure 11 for three components of a rock mass; intact rock, a clean joint and a high conductivity fracture.

\subsection{Hydraulic characteristics of rock joints}

It is well established that all intact rock, rock structures and rock masses are deformable. Figure 12 shows typical behaviour of fractures under normal and shear stress and the equivalent impacts on the hydraulic responses. As normal stress increases the closure of the defect decreases, Figure 12(a). Under increasing normal load there is a reduction in the transmissivity (hydraulic conductivity) of the fracture, but there is a residual transmissivity that indicates that at high normal stress levels the fluid flow is dominated by tube like channel flow, Figure 12(b). As expected this effect is size dependent because as the sample size increases there is an increased chance of intersecting more of the flow channels.

In relation to rock fractures, initially these respond to changes in normal effective stress $\left(\sigma_{\mathrm{n}}{ }^{\prime}\right)$ and shear stress $(\tau)$ elastically, Figure 12(a), with the magnitude of the opening or closure of the structure dependent on the normal stiffness of the fracture $\left(\mathrm{k}_{\mathrm{n}}\right)$. In a similar manner the shear displacement of the fracture depends on the shear stiffness $\left(\mathrm{k}_{\mathrm{s}}\right)$, Figure 12(c). Fluid flow in fractures takes place in the void space between the two sides of the fracture and all fractures have some irregularities. Hence even at high normal pressures these fracture surfaces are in irregular contact. 


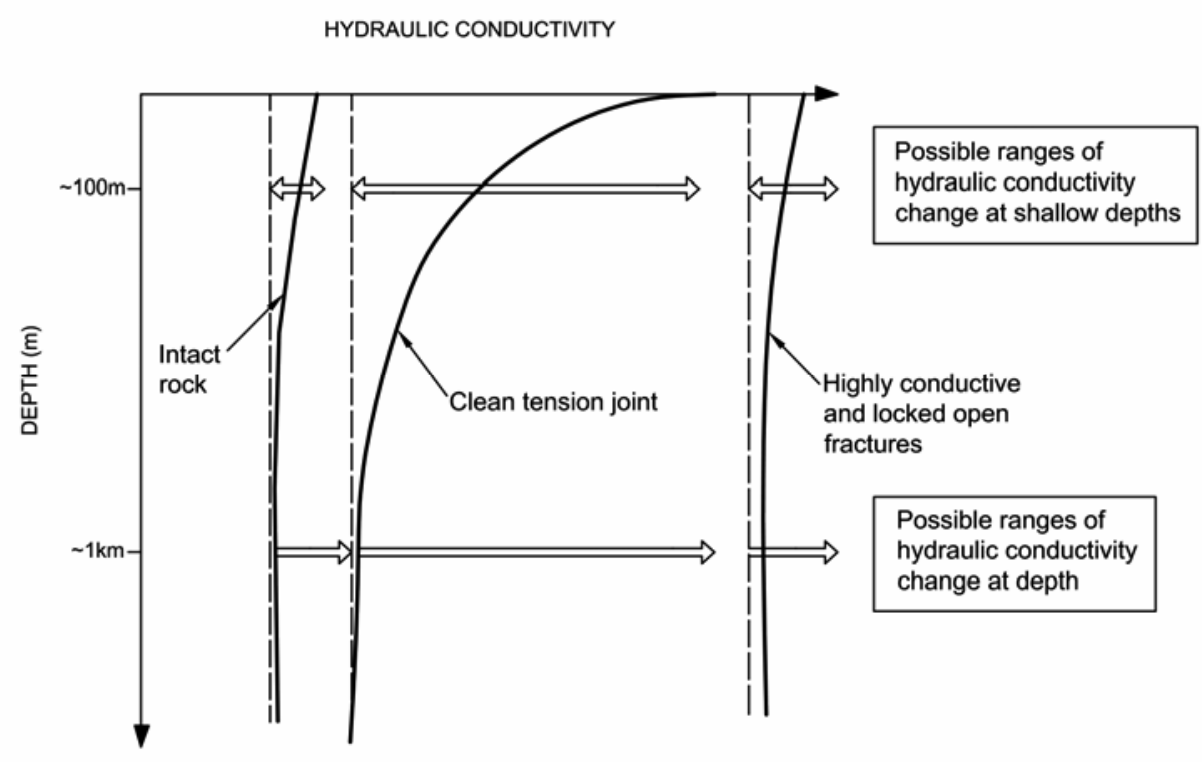

Figure 11 A schematic representation of the possible change in hydraulic conductivity in a rock mass for three components; intact rock, a joint and a high conductivity rock fracture. These possible changes are indicated for two conditions, shallow and deep (after Rutqvist and Stephansson, 2003)

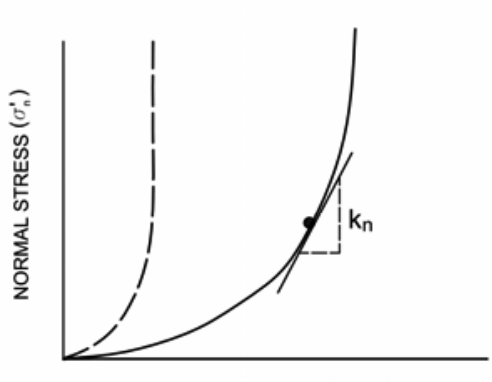

DEFECT CLOSURE $\left(\Delta \varepsilon_{n}\right)$

(a)

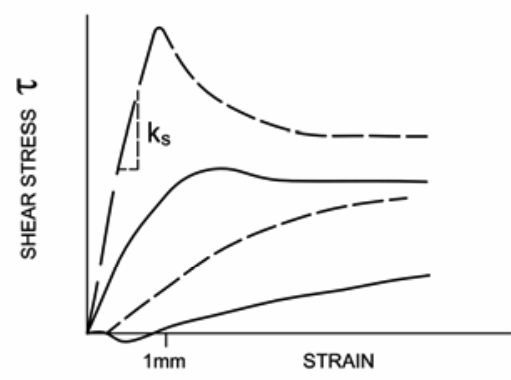

(c)

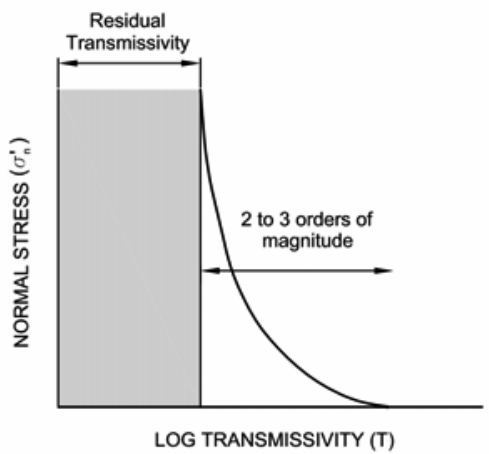

(b)

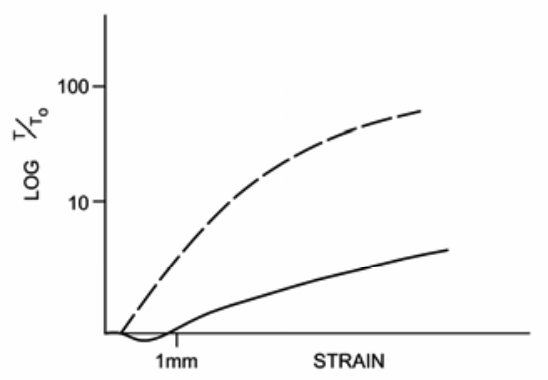

(d)

LEGEND

- - Drill Core

Insitu $\left(\mathrm{Im}^{2}\right)$

Figure 12 Typical deformation and hydromechanical response for a defect under normal (Figures a and $b$ ) and shear stress (Figures $c$ and $d$ ). Where $T=$ Transmissivity, $k_{n}=$ fracture normal stiffness and $k_{s}=$ fracture shear stiffness (after Rutqvist and Stephansson, 2003) 
During shear, tests have shown that joint dilation (hence aperture) starts to increase after small displacements $(>1 \mathrm{~mm})$. This has been shown to result in an increase in hydraulic conductivity of about one to two orders of magnitude up to displacements of about $5 \mathrm{~mm}$, Figures 12(c) and 12(d). However the impact on hydraulic conductivity of the fracture is confused by the development of gouge during shearing and the damage to asperities. It is generally accepted that whether or not hydraulic conductivity of the fracture increases or decreases depends on the properties of the rock, the properties of the defect surface and the stress applied. Once peak shear displacement is reached the relationship between aperture and hydraulic properties tends to breakdown.

The equation for laminar fluid flow in a fracture is given by (Wyllie and Mah 2004; Snow 1965 and 1968):

$$
K=\frac{g e^{3}}{12 b v}
$$

Where: $\quad K=$ Hydraulic Conductivity.

$e=$ Joint Aperture.

$b=$ Joint Spacing.

$v=$ Viscosity of water.

Although there has been conflicting evidence regarding the "cubic law", it has been found to hold true for a wide variety of factors (Rutqvist and Stephansson, 2003; and Louis, 1974). Various experimenters have demonstrated it applies over the general range experienced in most engineering situations $\left(\sigma_{\mathrm{n}}<10 \mathrm{MPa}\right)$. However there are some examples where the "cubic law" breaks down. Hence the law generally applies except under high stresses and with very fine fractures, aperture less than $50 \mu \mathrm{m}$ (Rutqvist and Stephansson, 2003).

For fully infilled structures the hydraulic conductivity is equal to the hydraulic conductivity of the infill, which will be higher than the rock mass (Louis, 1974).

Because the hydraulic conductivity of rock defects is proportional to the third power of the aperture, small changes in stress will result in quite significant changes to the hydraulic conductivity of most structures, refer to Figure 6.

Figure 13 illustrates the relationship between aperture, defect spacing and hydraulic conductivity for a parallel array of smooth joints. This is a very useful figure and can be used to obtain an order of magnitude estimate of likely hydraulic conductivity or defect aperture in many rock masses. This figure also illustrates that aperture is more significant than defect spacing for hydraulic conductivity, doubling the aperture has the same effect as increasing the number of defects by a factor of 10 .

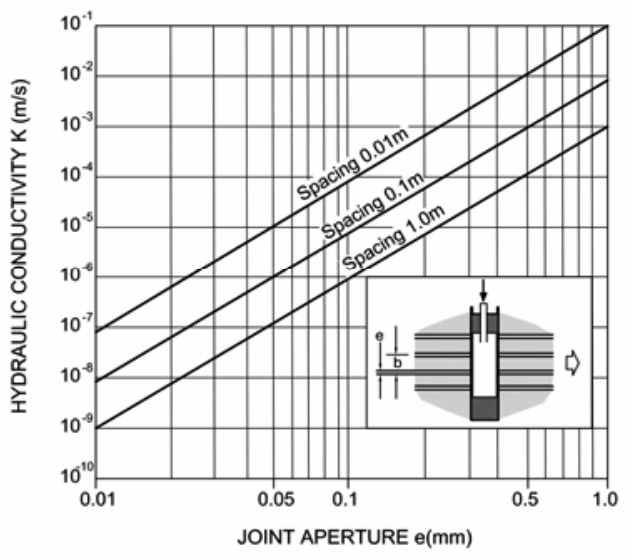

Figure 13 Hydraulic Conductivity of a set of parallel smooth joints for range of joint aperture and spacing (after Wyllie and Mah, 2004) 


\section{Pit slope deformations and failures}

\subsection{Pit slope movements}

Experience has shown that most pit slope failures pass through four stages of movement (Sullivan, 1993):

1. Viscoelastic response.

2. Creep.

3. Cracking and dislocation.

4. Collapse or failure.

The movements that occur prior to collapse can result from multiple phenomena including: elasto-plastic deformations, viscous deformations, softening, failure of the rock mass, slip on structures and progressive failure. Cracking and dislocation of the slope are differentiated in this classification because of the potential hydromechanical impacts and also because experience has shown it is usually possible to undertake remedial measures to stabilise the slope even at that stage. Although failure in an engineering sense, including civil engineering, is usually classified as Stage 3, this is inadequate in mining because it is often possible to either continue mining and or undertake remedial measures that avoid the slope actually collapsing. Hence the adverse economic loss may be avoided at least in large part.

According to Fell et al. (2000) all the first three stages of these movement creep; differentiated into Primary, Secondary and Tertiary; where Primary creep occurs at a decreasing strain rate, Secondary creep occurs at a constant strain rate and Tertiary occurs at an increasing strain rate, Figure 14.

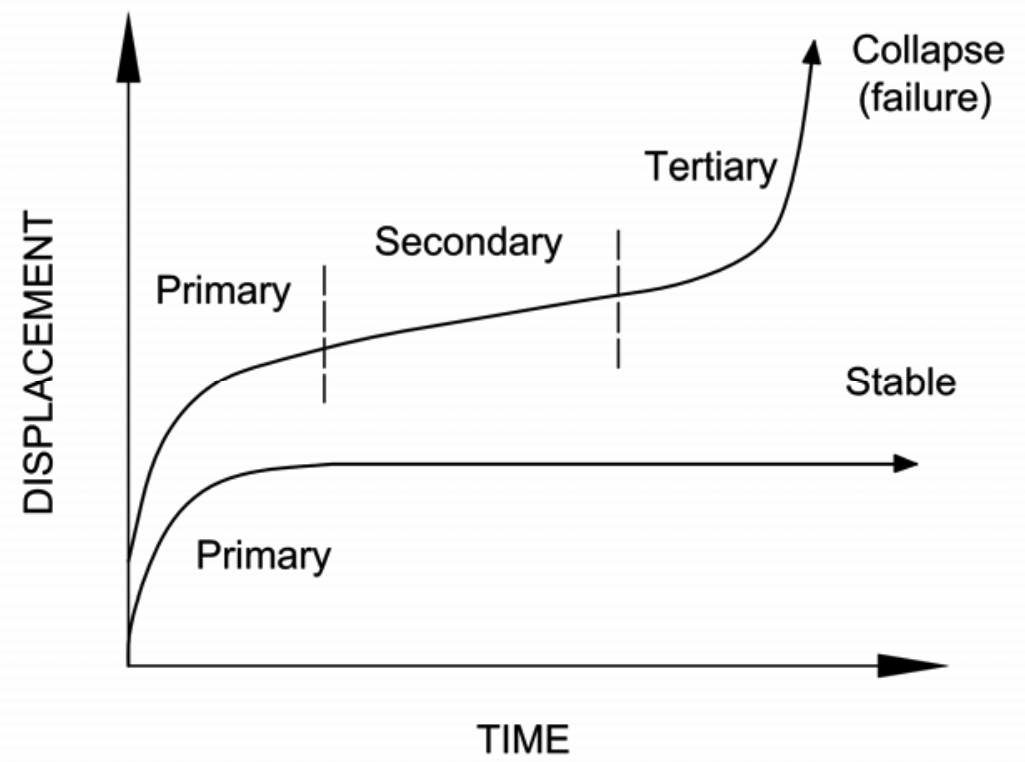

Figure 14 Diagrammatic representation of creep stages for moving slopes (after Fell et al., 2000)

Zavodni (2001) has termed the initial phase of movement experienced by all pit slopes, the "Initial Response". This "Initial Response", which he notes is time dependent, includes elastic rebound, relaxation and/or dilation of the rock mass. The "Initial Response" probably includes Stage 1 and at least part of the Stage 2 movements described above.

Creep movements vary with the stress conditions in the slope. As pore pressure in the slope increases due to rainfall the effective stress in the slope reduces, the stress path moves closer to the peak strength envelope, Figure 3, and the creep rate increases.

However, the author has found it more useful to try and separate Primary and Secondary creep movements (Figure 14), because the former decreases with time, while the latter are more probably associated with some 
yielding or strength loss of the rock mass and as such are important for long term management of slopes. Experience has shown it is usually possible to distinguish the early part of slope movements related to excavation from the subsequent secondary creep phase. The identification of a separate secondary creep phase also has importance for the hydromechanical coupling of the rock mass.

\subsection{Deformation of pit slopes and hydraulic impacts}

The viscoelastic movements are highly variable and depend on the geotechnical character of the rock mass, with stiff high strength rock masses deforming the least and low modulus low strength rock masses deforming the most. Although experience shows it has been quite difficult to measure these movements in many cases because of mining activities, total movements recorded for the "Initial Response" stages are in the range 100 to $1000 \mathrm{~mm}$, Zavodni (2001) and Sullivan (1993).

This initial stage of movement has great significance for the hydrology of the rock mass, because mining changes the stresses in the rock mass resulting in movement which changes the water pressure and the hydraulic parameters; hydraulic conductivity and storage. This is Type 1 and Type 3 hydromechanical coupling.

It is now common for many large open pits to undertake deformation modelling as an input to dewatering and depressurisation studies. This modelling attempts to predict these initial phases of pit response to excavation. Figure 15 shows an example of modelling for an $835 \mathrm{~m}$ deep open pit. The geology comprises horizontally bedded sediments overlying granitic bedrock. Both rock masses are high strength, broadly jointed and with a high in situ horizontal stress.

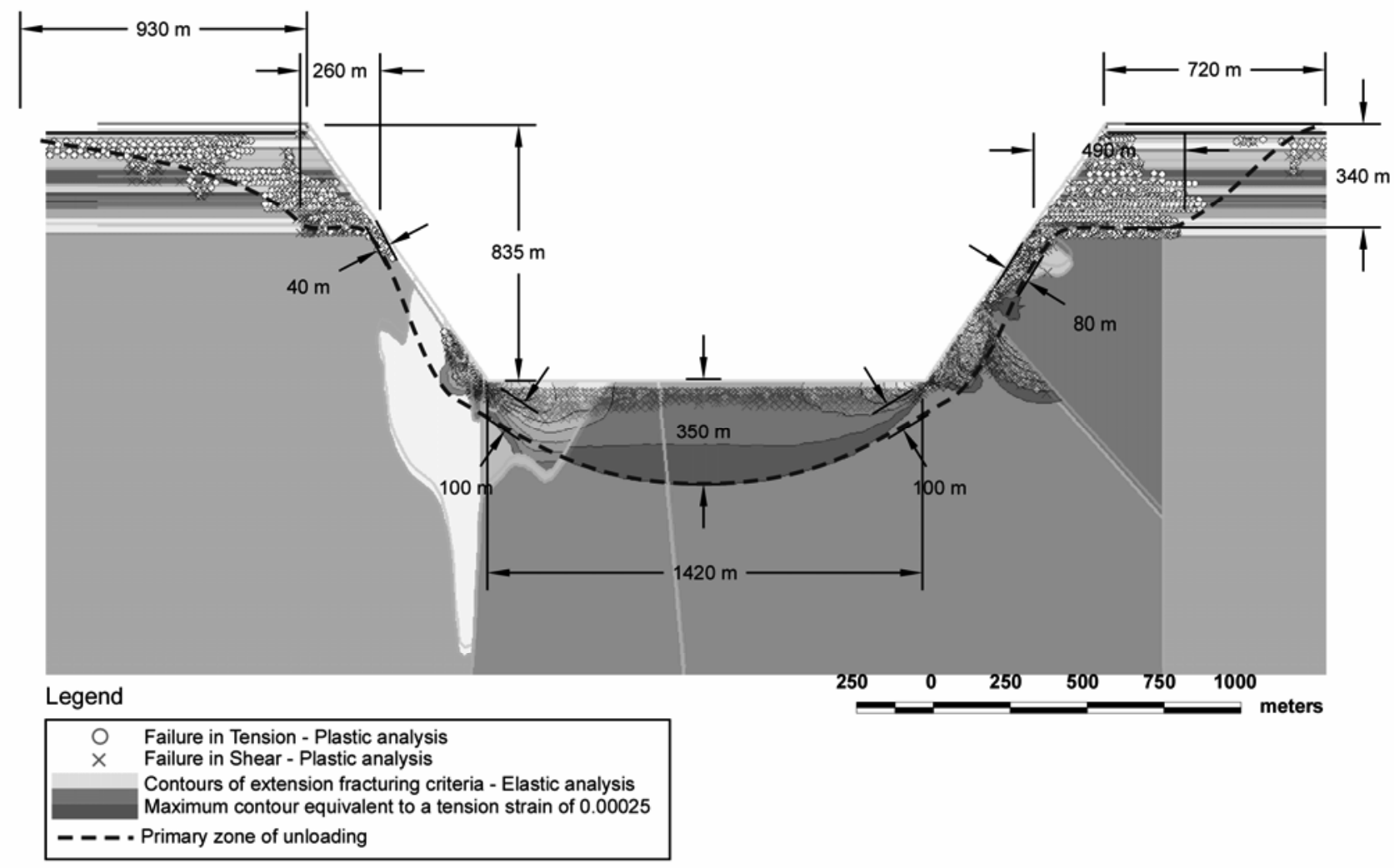

Figure 15 Numerical deformation modelling for a deep open pit to assess the effects of unloading resulting in strains in the rock mass. The geology consists of a thick sequence of bedded sedimentary rocks overlying basement rocks. The modelling utilised the concept of extension strain (Stacey et al., 2003) 
The modelling uses the concept of limiting extensional strain as the rock mass deformation criteria, Stacey et al. (2003). This criteria has been utilised to define the primary zone of "unloading" effects due to mining. The movements are very extensive horizontally in the sediments and more closely associated with the pit wall in the granite. There is also a very extensive zone of movement developed for a depth of $350 \mathrm{~m}$ below the pit floor. Similar movements have been reported for actual excavations by Burland et al. (1977). O'Brien and Sullivan (1988); and Small and Morgenstern (1992) and Zavodni (2001). In each case the style and magnitude of the movements was related to the geology and geotechnical properties of the mass.

The effect of these deformations on hydraulic properties of the rock mass can be quite substantial. Wyllie and Mah (2004) reported an 18 fold increase in hydraulic conductivity associated with a $170 \mathrm{~m}$ deep excavation in granite for the Three Gorges Dam project in China.

\subsection{Movements, mechanics and failure of natural slopes}

Leroueil et al. (1996) developed a geotechnical classification system for describing the movements of natural failures and incorporated the concepts of peak and residual strength, Figure 16. The system recognized that once significant movement has occurred (failure or collapse) then the strength is at or close to residual. In this state less energy is required to re-initiate movement and hence the slope becomes more sensitive to external environmental factors.

Couture and Evans (2006) recognised that large scale slope movements and failures are not rigid bodies and usually comprise both a number of components and mechanisms. This is illustrated diagrammatically in Figure 17 for a typical large natural landslide. It is evident that the controlling mechanics of the slope and the mechanisms controlling behaviour change substantially pre and post failure. Although used in part to explain the different behaviours in different parts of the slide the explanation is a useful aid for understanding some of the behaviours observed in open pits. The mechanism in Figure 17 started with the initial failure defined in rock mechanics as structurally controlled, progressed through soil mechanics for a particulate aggregate of grains and finally entered the field of fluid mechanics to explain the "flow" characteristics and large post failure deformations. This process is associated with large increases in the water content of the mass and very large increases in the rates of movement, Figure 17. It is evident from Figure 17 that the type of hydromechanical coupling also changes from Type 1 to 4.

The other really important factor highlighted by Figures 16 and 17 is the equal importance placed on all stages of the landslide from initial creep movements prior to failure (Figure 16) to the deformations post failure (Figure 17).

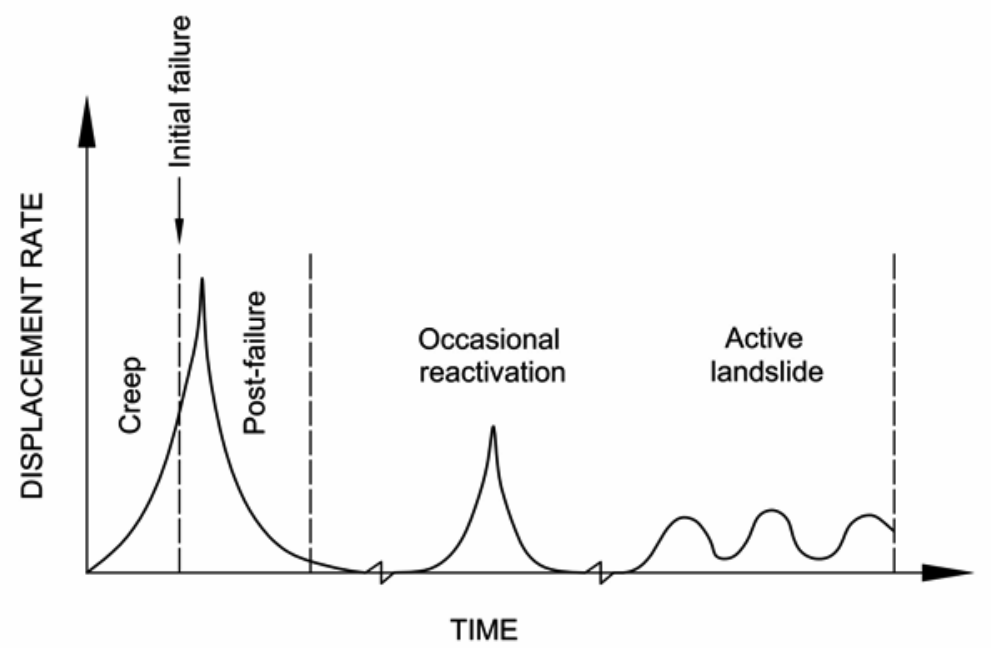

Figure 16 Diagram showing different stages of slope movements for a natural landslide (after Leroueil et al., 1996; and Fell et al., 2000) 


\section{MECHANICS GOVERNING BEHAVIOR}

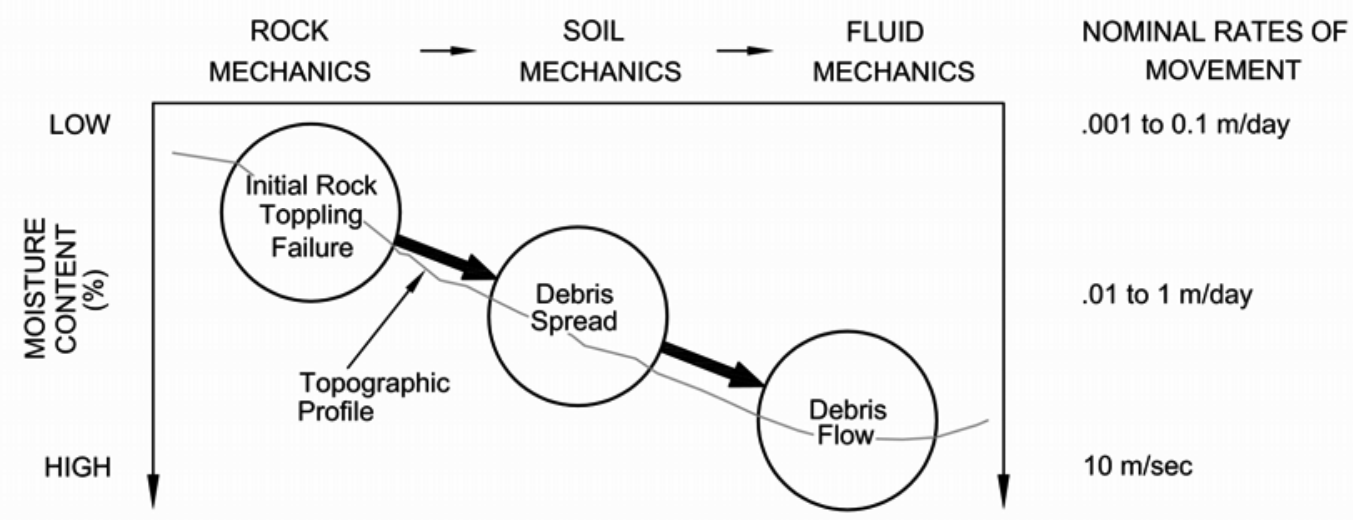

\section{Figure 17 Flow chart illustrating the mechanics for a large natural landslide. Also shown are the general relative moisture contents and indicative movement rates. The light grey line represents the topographic profile of the slope}

\subsection{Categorisation of pit slope failure movements}

Broadbent and Zavodni (1982) and Zavodni (2001) used empirical evidence to categorise pre-failure pit slope movements, Figure 18. The forerunner to this system was in part the classic case study of slope failure monitoring and prediction by Kennedy and Niermeyer (1970) for Chuiquicamata Mine. The simple movement concept of continuous accelerating movements leading to failure still forms the basis of understanding and performance prediction used by many practitioners.

Initially three basic types of pit slope failure patterns were identified by Broadbent and Zavodni (1982), Figure 18:

- Regressive.

- Progressive.

- Transitional.

Although very powerful as an educative tool, the classification system of Broadbent and Zavodni (1982) is limited because:

- It fails to show the very large time range over which the acceleration stage of the movement can take place, which can vary from minutes to years.

- Implicitly it indicates there is always a long time period of movement and large displacement prior to failure.

- There are other movement patterns not captured by these models.

The emphasis in slope monitoring in open pits has traditionally been on the prediction of the movements leading up to failure, Stages 3 and 4. However for most pit slope situations this emphasis, while very important, is inadequate because firstly it presupposes that a failure will declare itself early with a clearly evident period of acceleration and secondly it is often the early part of the slope movement curve that is the most important for managing the slope. This early part of the curve represents the period before complete cracking and dislocation have occurred. Hence it is of economic importance because in this period it is often possible in the mining context to undertake remedial measures that mitigate the failure impacts and hence avoid significant economic loss. The other important reason is that during this period of movement there is an increased sensitivity to environmental effects and an increased potential for a significant hydromechanical coupling event. 


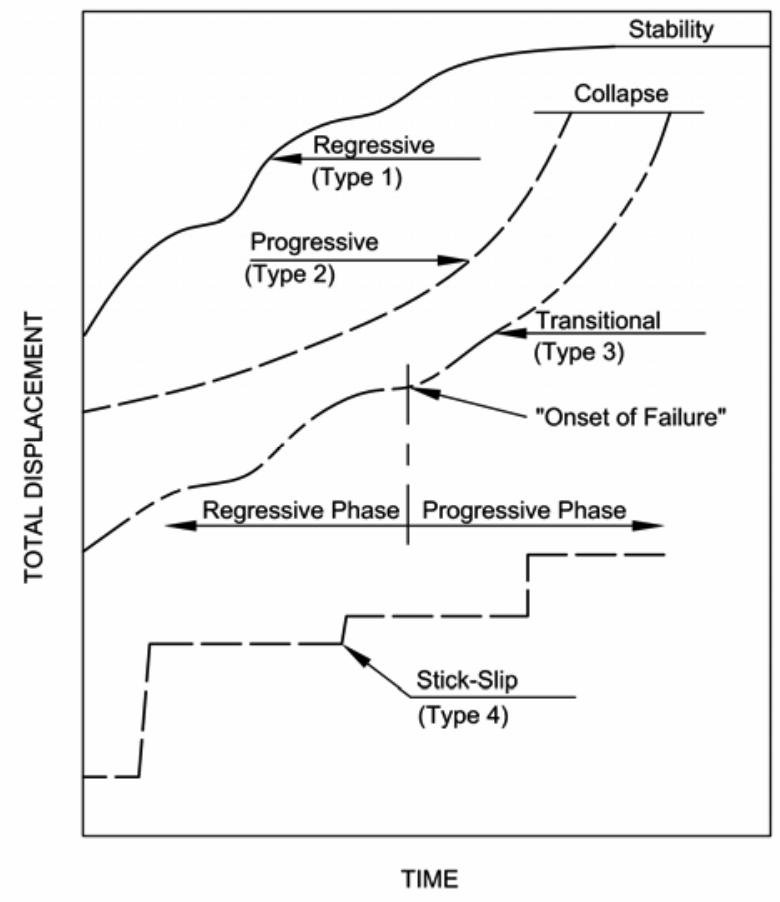

Figure 18 Typical time displacement curves for pit slope failures (after Broadbent and Zavodni, 1982; and Sullivan, 1993)

It is the authors experience with 14 inter ramp scale failures in high strength shale slopes that included polished bedding planes and undulating faults dipping sub-parallel to parallel and partially undercut by the pit slope that:

- Failure occurred after very small total movements, 20 to $55 \mathrm{~mm}$ and 225 to $395 \mathrm{~mm}$.

- Long term movement rates were in the range 0.025 to $0.17 \mathrm{~mm} /$ day.

- Detailed monitoring was available for four failures and three of these cases showed no acceleration prior to failure.

- In many cases there were no other visible signs of imminent failure.

- Failures were quite sudden.

The author, Sullivan (1994) also reported experience with long term monitoring of a cable bolted shale footwall that over a period of six years of movement had a total displacement of less than $0.3 \mathrm{~m}$. Displacement rates were in the range 0.1 to $0.3 \mathrm{~mm} /$ day and the slope appeared to be stabilising immediately prior to failure.

Post failure behaviour and movements have received scant attention. This is in part a function of the practical reality of mining because when slopes fail the area has usually been cleared, the failure usually falls onto the pit floor then is cleaned up. However more recently there have been a number of tragic pit slope failures, which have undergone "liquefaction type failures" displaying very large post failure deformations of the failed mass.

Consequently, if the aims of a pit slope movement classification system are to illustrate the various movement patterns that a failure could be expected to display, then it is essential to also show that there is a class of failures that do not simply accelerate to failure and then after collapse stop. These "liquefaction type failures", which display very large post failure deformations and high velocities reach a stage where the mechanisms controlling the movements and the engineering principles change, moving out of the realm of rock mechanics and into fluid mechanics, Figure 17. 
These latter examples also highlight the fact that part of the essential role of the geotechnical engineer is not only to manage and predict failure but to accurately predict the behaviour and movements post failure.

So we now need to add a fifth component to the possible stages of pit slope movements that could be experienced:

- Elastic.

- Creep.

- Cracking and dislocation.

- Collapse (failure).

- Post failure deformation.

These stages are illustrated schematically in Figure 19 for a typical failure, as a plot of velocity versus time. This figure illustrates the number of different failure paths that typical pit slope failures may follow. The emphasis in this figure is on differentiating between failures which simply stabilise and those that show large post collapse velocities.
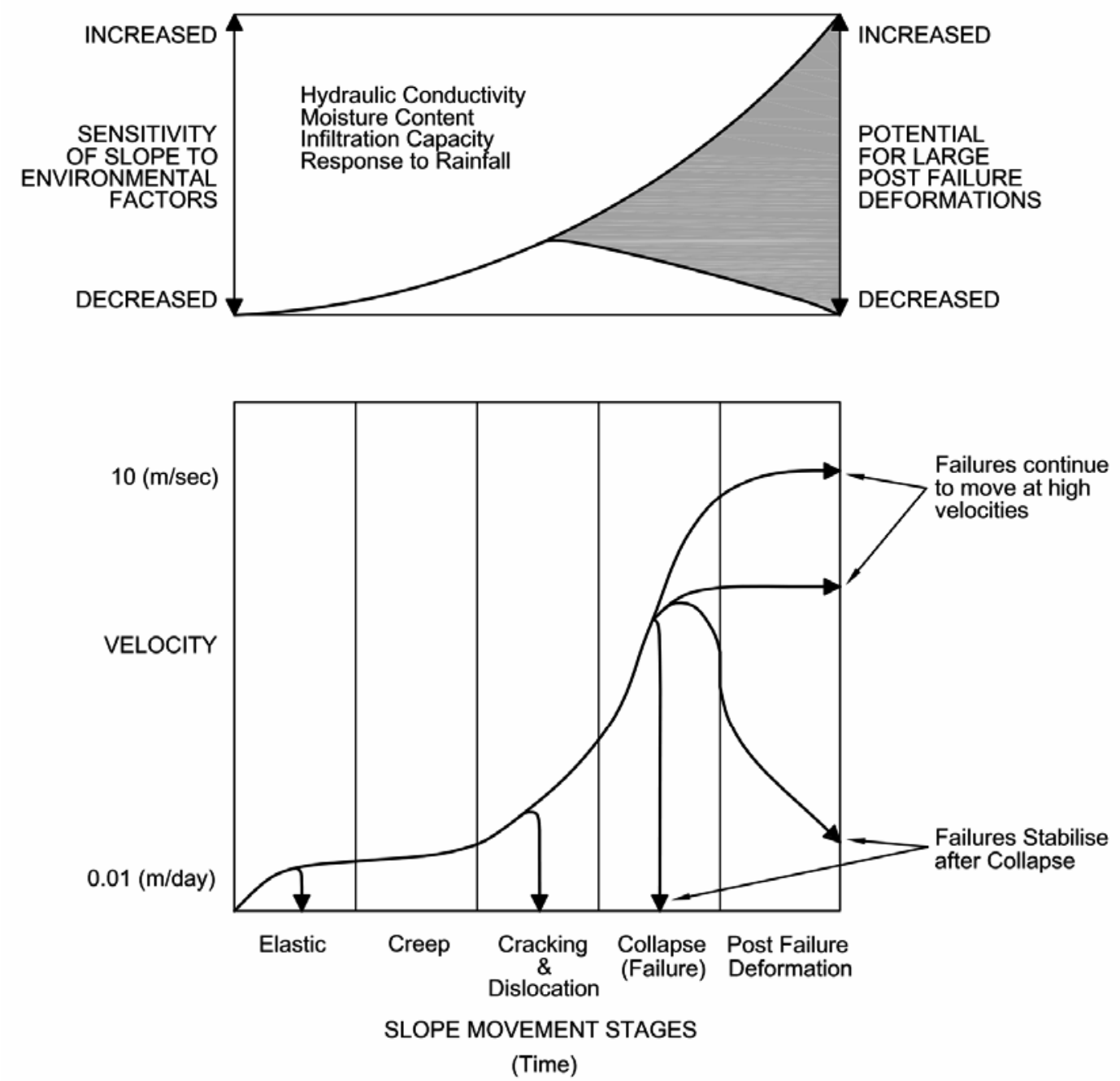

Figure 19 Schematic illustration of the five principal stages for a pit slope failure and the range of post collapse velocities. Also illustrated are the changes in hydraulic properties over time and the implications of these changes on the potential for large post failure deformations 


\subsection{New pit slope movement classification system}

As pit slope management systems have matured, the sophistication and accuracy of monitoring equipment has increased; and the body of empirical evidence has grown, an improved understanding has developed. In parallel there has been an increased focus on the earlier pre-acceleration stages of slope movement, Stages 1 to 3 .

Furthermore, in order to better explain the full range of movements experienced by pit slopes and to capture the potential for catastrophic movements post failure it is necessary to divide pit slope movements into three periods; pre-failure movements, failure movements and post-failure movements. This system has three benefits, it allows a more complete description of the wide range of movement types that could occur leading up to the clear onset of failure movements; it allows the wide range in failure acceleration periods to be indicated and it also allows the range of post failure deformations behaviours to be illustrated, including the velocity.

Published records and the authors experience has led to recognition of the following types of pre-failure movement patterns; Broadbent and Zavodni (1982); Sullivan (1993) and Zavodni (2001); Figure 20:

- Linear.

- Bi-linear.

- Stick-slip.

- Regressive.

- Transitional.

- Slow accelerating.

- Linear accelerating.

- Accelerating (Progressive).
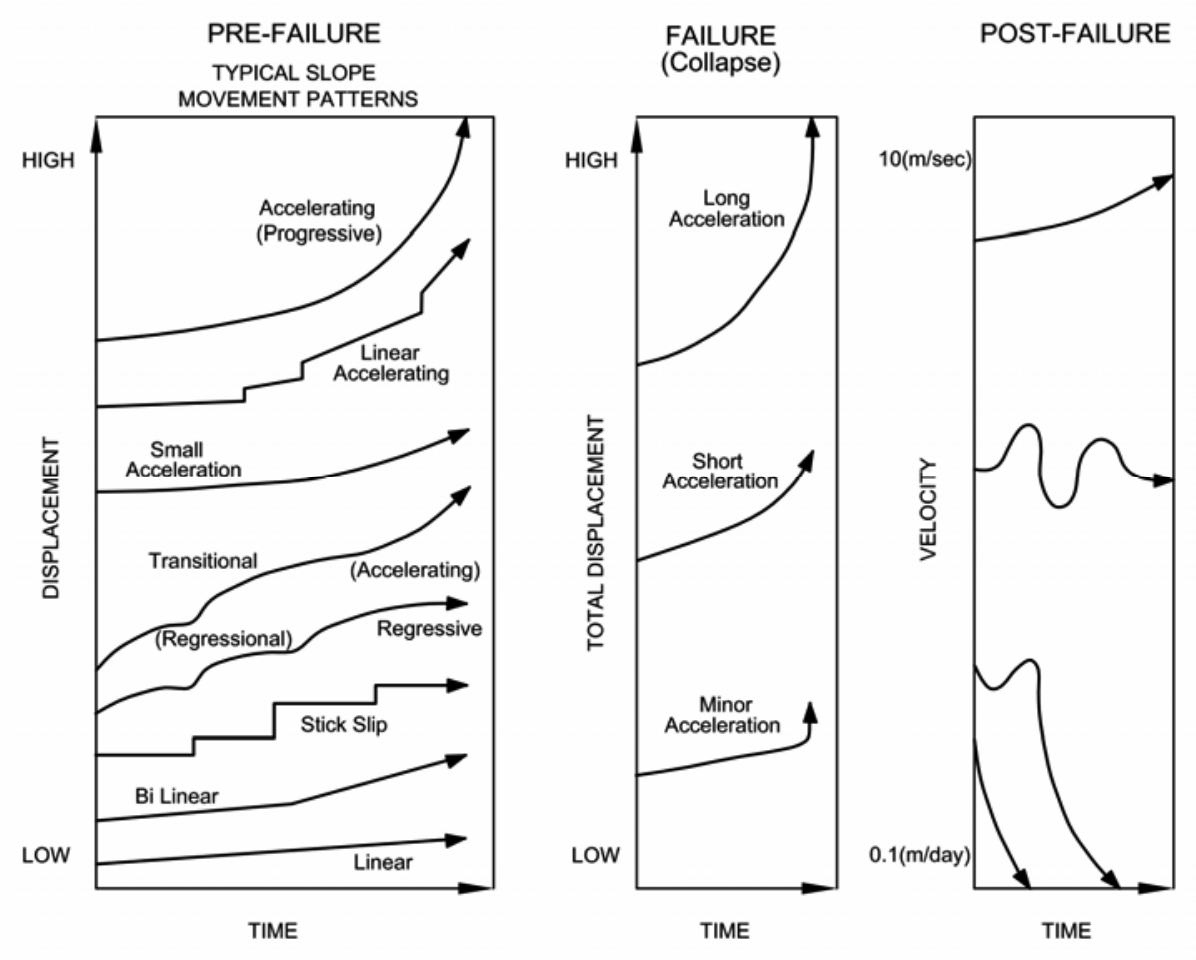

Figure 20 Breakdown of pit slope failures into three periods of movement — pre-failure, failure and post failure 
In the period leading up to and immediately prior to failure three types of general behaviour have been observed by the author, Figure 20:

- A long period of continuous acceleration with movements best described by a Power Law. This period is usually measured in weeks to years.

- A shorter period of acceleration with a lower total displacement prior to collapse and usually measured in days to weeks.

- Failures that occur over very short periods and usually with minimal acceleration observed prior to collapse with displacement measured in minutes to days.

The post failure period is illustrated with three examples, Figure 20:

- High velocity failures of the order of $10 \mathrm{~m}$ per second.

- Failures that show moderate and cyclic velocities post failure.

- Failures that stop after collapse with minimal further movement.

\subsection{Rates and scales of movement}

It is the author's experience that velocity is one of the key parameters for predicting future performance.

Glastonbury (1999) analysed a number of published records for pit slope failures and developed the relationship shown in Figure 21, between the period of accelerated movements, Tertiary creep (Figure 14), and the displacement rate at that onset of the Tertiary creep. This correlation is useful because it shows:

- The creep rate at the end of the secondary creep phase varies by about two orders of magnitude, from 0.1 to $10 \mathrm{~mm} /$ day.

- The period of the accelerating phase leading up to failure, Tertiary creep, varies from days to years.

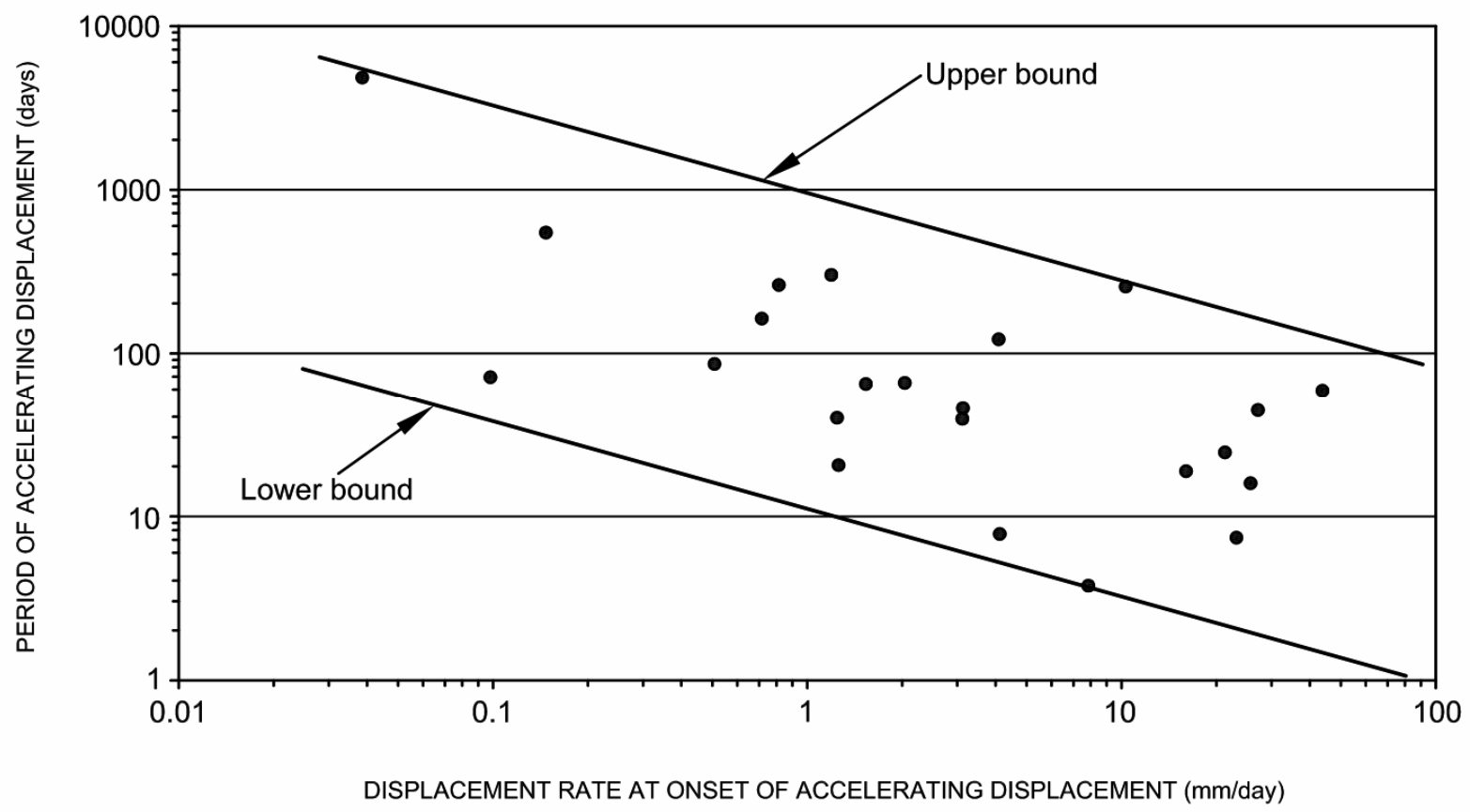

Figure 21 Relationship between the displacement rate at the onset of Tertiary creep and the duration of Tertiary creep (after Glastonbury, 1999) 
The relationship inferred by Glastonbury (1999) is an inverse relationship, where a lower initial displacement rate correlates with an increased period of Tertiary creep leading up to failure. The author has experience with other pit slope failures that do not fit this relationship, where failures occur after small total strains and short periods of acceleration, even though the initial displacement rate is very small. These failures were of moderate size and occurred along structures with a brittle stress displacement curve that also showed a large difference between peak and residual shear strength.

A very important finding of Glastonbury (1999) for practitioners is the observation that similar rock masses and failure types would tend to fit in the same section of the graph. To most experienced practitioners this makes sense.

Many authors have attempted to set limits on scales and rates of movement leading up to pit slope failure, based on individual experience and published records including; Broadbent and Zavodni (1982); Martin (1993); Sullivan (1993); Fell et al. (2000) and Zavodni (2001).

What these attempts illustrate is that there is no one easy answer. Intuitively given the various characteristics of rock masses and the ranges in properties for each parameter it makes sense that there must be a wide range of potential movement patterns that slopes may demonstrate leading up to failure. Notwithstanding this, limits or threshold values based on empirical evidence are extremely useful tools for educating judgement. It is clear from the author's experience that attempts at defining the bounds of possible or expected behaviour are a valuable aid for the practicing engineer.

Table 1 presents a summary from the author's experience and some collated experience of others on the threshold velocities for planning. This table is weighted towards the early lower velocity movements and this is deliberate because in the author's experience one of the most important parts of the pit slope management is to be able to predict at an early stage what the future performance is likely to be.

Table 1 Classification of pit slope velocities for planning

\begin{tabular}{|c|c|c|c|c|}
\hline \multirow[t]{2}{*}{ Movement Class } & \multicolumn{2}{|c|}{ Velocity } & \multirow[t]{2}{*}{ Comment } & \multirow{2}{*}{$\begin{array}{c}\text { Sensitivity to } \\
\text { Environmental Factors, } \\
\text { e.g. Blasting, Rainfall } \\
\text { Runoff, etc. }\end{array}$} \\
\hline & (mm/day) & (mm/month) & & \\
\hline Low creep & $<0.05$ & $<1.5$ & $\begin{array}{c}\text { Also possibly Stage } 1 \\
\text { movements }\end{array}$ & Very low \\
\hline Significant creep & $0.05-0.1$ & $1.5-3$ & $\begin{array}{c}\text { Also possibly Stage } 1 \\
\text { movements }\end{array}$ & Low \\
\hline $\begin{array}{l}\text { Definite movement of } \\
\text { slope related to shear } \\
\text { or displacement on } \\
\text { structures }\end{array}$ & $0.1-0.25$ & $3-7.5$ & $\begin{array}{c}\text { Could stabilise if strain } \\
\text { hardening }\end{array}$ & Medium \\
\hline $\begin{array}{l}\text { Likely to fail } \\
\text { sometime in the future }\end{array}$ & $0.25-0.5$ & $7.5-15$ & & Medium to high \\
\hline High chance of failure & 1.0 & 30 & & Medium to high \\
\hline $\begin{array}{l}\text { Pre-failure collapse } \\
\text { movements }\end{array}$ & $>1.0$ & $>30$ & & High \\
\hline
\end{tabular}

Table 2 is a tabulation of the various critical velocities proposed by various practitioners for predicting when failure could be expected in a short time. The range of velocities is very large and is probably a reflection of the range of conditions experienced with slopes of various scales, displaying various failure mechanisms and in rock masses with a very wide range of geotechnical properties. 
Table 2 Classification of pit slope velocities for determination of the critical velocity when failure could be expected in a short time

\begin{tabular}{lcc}
\hline Author & $\begin{array}{c}\text { Velocity } \\
\text { (mm/day) }\end{array}$ & $\begin{array}{c}\text { Period over which Velocity Applies } \\
\text { (days) }\end{array}$ \\
\hline Ryan and Call (1992) & 12 & 2 \\
Zavodni (2001) & 50 & 2 \\
Zavodni (2001) & 17 & 2 \\
Martin (1993) & 15 & 2 \\
Zavodni and Broadbent (1982) & $10-100$ & \\
Zavodni (2001) Borax Mine & 50 & \\
Call and Nicholas (from Zavodni 2001) & 150 & \\
Savely (1993) & 300 & $<$ hours \\
Sullivan (1993)* & $30-1000$ & \\
\hline
\end{tabular}

Note: * Minimum instantaneous velocity immediately prior to collapse.

\subsection{Potential for hydromechanical coupling}

Figure 19 shows the velocity over time for a typical failure with a number of alternate outcomes. Experience has shown that many slopes and failures may show no further movement after either initial elastic response, cracking and dislocation and/or collapse (Figure 19).

As illustrated in Figure 19 those slopes that progress past the elastic stage will have an increased sensitivity to environmental factors. This sensitivity will also increase with displacement.

The physical factors that could change as the slope progresses through the stages are:

- Hydraulic conductivity - increasing.

- Moisture content - mainly increasing but depending on the environment.

- Infiltration capacity - increasing.

- Response to rainfall runoff - increasing.

Depending on a number of site specific features this could lead to an increased or decreased potential for large post failure deformations, liquefaction type failure.

\section{Assessment of depressurisation, pore pressures and stability for different scales of slopes}

\subsection{Introductory points}

For many mine slopes it is very difficult to model the piezometric conditions and the pore pressure response with a high degree of accuracy. This is because the pit slopes have only recently been excavated, the hydrogeological regime has not reached equilibrium with the prevailing environment; the geology is both complex and variable; and lastly the areas over which conditions are predicted are usually very large.

In mining an essential element is to understand the geological environment and what this might mean hydrogeologically in order to plan an effective monitoring program and to interpret the results. One of the other keys is to understand the hydrogeological response and the hydromechanical coupling of rock masses caused by the mining cycle. 
In order to establish a reliable hydrogeological model it is usually necessary to instrument the slopes and monitor them over a long period, to establish the relationships between rainfall, runoff, drainage, dewatering and depressurisation measures.

\subsection{Depressurisation}

In mining we are usually restricted to one of five techniques for depressurisation of pit slopes:

- Natural Drainage.

- Horizontal Drains.

- Pumped Wells.

- Drainage adits.

- Unloading.

Brown (1982) undertook an evaluation of these methods and their applicability to pit slope depressurisation and used the Coefficient of Consolidation $\left(c_{v}\right)$ and equation (3) to illustrate the general ranges within which each technique will be effective. This is illustrated in Figure 22 for a range of general soil and rock mass types. This chart together with equation (3) and Figure 13 are very useful for providing approximate assessments of the applicability of various depressurisation measures.

\begin{tabular}{|c|c|c|c|c|}
\hline \multicolumn{5}{|c|}{ MAIN MATERIAL TYPE IN SLOPE } \\
\hline SOILS: & CLAYS & SILTS & \multicolumn{2}{c|}{ SANDS } \\
\hline ROCKS: & $\begin{array}{c}\text { HYDROPHYILLIC } \\
\text { CLAY RICH ROCKS }\end{array}$ & $\begin{array}{c}\text { ALTERED ROCK MASS } \\
\text { WITH INCREASING } \\
\text { CLAY CONTENT }\end{array}$ & $\begin{array}{l}\text { WEATHERED } \\
\text { ROCK MASS }\end{array}$ & $\begin{array}{c}\text { CLEAN } \\
\text { JOINTED } \\
\text { ROCK MASS }\end{array}$ \\
\hline
\end{tabular}

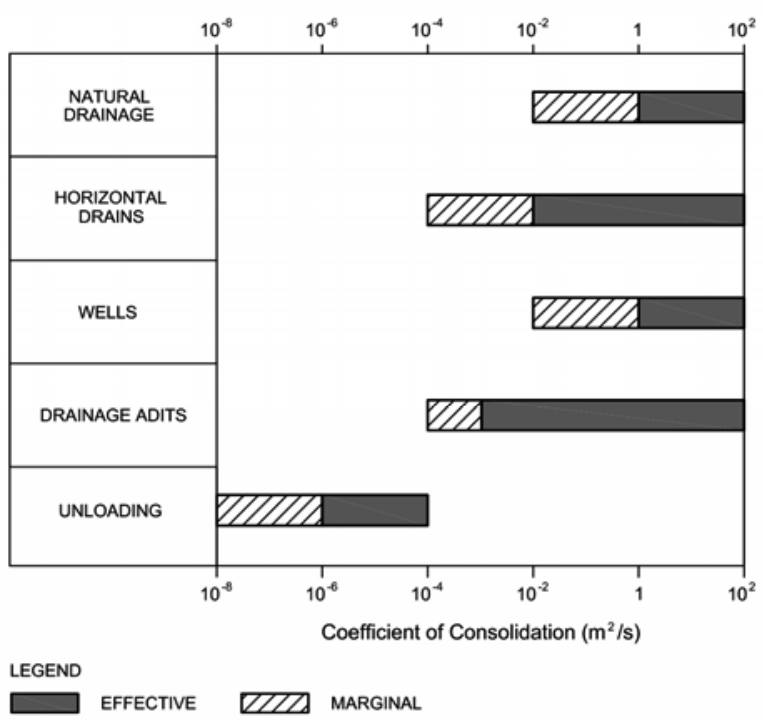

Figure 22 Methods and applicability of dewatering and depressurisation techniques (after Brown, 1982)

Although rocks are considered a discontinuous medium due to the presence of rock structure, most problems of depressurisation for large open pit slopes are usually handled by assuming the discontinuous rock mass may be adequately represented by a representative continuum like a porous granular media. This approach yields acceptable results where the structural fabric is small in relation to the slope scale. However if the structure density is low or a few specific major structure(s) are involved in a stability question, then groundwater conditions and or flow in and around the individual structures need to be considered. 
Uncoupled or partially coupled analyses of flow are the dominant approaches in hydrogeology and most texts either ignore the hydromechanical coupling altogether or only cover it in passing. Fully coupled analyses of fluid flow and deformation are now becoming more common in geotechnical studies and generally handled numerically (Pariseau, 2001). However the application of rigorous modelling of hydromechanical coupling successfully to pit slopes is often limited in part by our ability to adequately categorise the rock mass due to the inherent heterogeneity of the geology. This is particularly the case before large scale exposures have been developed. Permeability is one example of the heterogeneity of the earth, it can range over five orders of magnitude, can change by orders of magnitude as a result of movement of rock masses and is sensitive to the scale of the measurement, Figures 10 and 12.

In addition groundwater models rely almost exclusively on mass balance and hence monitoring is essential for calibration in order to predict future performance.

\subsection{Estimating pore pressures and stability of slopes}

\subsubsection{Slope depressurisation}

For most new open pits it is very difficult to accurately predict pore pressures ahead of mining. Experience has shown it is usually necessary to rely on a general understanding of hydrogeology and if possible empirical evidence from other similar rock masses.

\subsubsection{Small slopes - individual blocks or mine benches}

Experience has shown that at the small scale in most rock masses, monitoring of pore pressures and prediction of performance is practically impossible. This is in part a function of the fact these slopes are:

- Often in the partially saturated zone and above the water table.

- The stability and Factors of Safety for these slopes are very sensitive to the input parameters.

- They are very sensitive to local rainfall runoff and impacted by transient water pressures.

- They are affected by local joint aperture and continuity.

- They are usually located within the de-stressed zone of the rock mass and affected by excavation practices.

For small slopes high transient pore pressures can occur with infiltration into cracks and open structures. In general these features are usually missed by most instrumentation. At this scale it is very difficult to make the correct assumptions and calculations are often misleading.

\subsubsection{Medium scale slopes - multiple bench to interramp scale}

Experience with medium scale slopes shows a high degree of variability in pore pressures even for piezometers that are close together. It is usually possible to undertake meaningful stability analyses using pore pressures interpolated from nearby piezometers and or observations. Although these are often modelled for a range of possible pore pressure distributions in order to effectively capture the uncertainties. In general these slopes may be just as complex as smaller scale slopes.

While this sounds straightforward, the reality is usually quite different because:

- Often only 24 hour rainfall data is available and shorter duration rainfall can be critical.

- The failure mechanisms usually involve a number of structures of different character and accurately determining the pore pressure distribution in each is difficult.

\subsubsection{Large scale slopes - interramp to overall scale}

Accurate prediction of pore pressures in very large slopes usually requires a mass water balance assessment calibrated using medium to long term responses in multiple piezometers installed at multiple elevations through the rock mass. 
In pit slopes the problems of interpretation and prediction can also be compounded by the effects of unloading on pore pressures.

Another complicating factor is that experience has shown that movement of very large slopes may occur over a wide range of Factors of Safety.

\subsection{Open pit groundwater conditions for analysis and design}

In most large deep open pit slopes we are generally dealing with four possible groundwater design conditions, Figure 9(a):

- Unsaturated conditions with no pore pressures above the water table.

- Transient saturated conditions in the upper levels with partial pore pressures due to transient rainfall infiltration response.

- Saturated conditions below the groundwater table with full hydrostatic pore pressures $\left(\mathrm{P}=1 / 2 \gamma_{\mathrm{w}} \mathrm{h}^{2}\right)$.

- Saturated conditions below the groundwater table with partial pore pressure $\left(\mathrm{P}<1 / 2 \gamma_{\mathrm{w}} \mathrm{h}^{2}\right)$. Less than hydrostatic, usually due to an artificial depressurisation system such as horizontal drains or drainage adit.

If the groundwater table is deep relative to the open pit and the rainfall is relatively high, then the zone over which Condition 2 could apply may be quite significant. Similarly many large open pit slopes are now dealing extensively with partial pore pressures below the groundwater table, Condition 4.

\section{Examples of hydromechanical coupling}

\subsection{Introduction}

This section of the paper presents examples of some different types of hydromechanical coupling observed in pit slopes. An attempt is also made to illustrate the main mechanisms that underlie the phenomena and to highlight the current gaps in the understanding of such mechanisms.

Hydromechanical coupling is the physical interaction between hydraulic and mechanical processes. Many if not most geotechnical processes associated with pit slope deformation and behaviour can only be fully understood by considering the coupling between fluids and the rock masses that contain them. The mechanical interactions that underlie and provide a unifying view of many geotechnical phenomena are generally not well appreciated.

In open pits hydromechanical coupling can be direct or indirect, where direct hydromechanical couplings occur through an interaction between the deformation of the slope and the pore-fluid, and indirect hydromechanical couplings occur when these two factors are not directly linked.

In situ stress measurement using hydraulic fracturing, which many geotechnical engineers are well aware of, is simply an example of hydromechanical coupling.

\subsection{Rock mass and structure and response to depressurisation}

Figure 23(a) shows a broadly jointed rock mass, which is intersected by some major thrust faults (see Figure 23(b)). The overall slope is some $200 \mathrm{~m}$ high at an angle of $40^{\circ}$ and this is the upper section of a planned 700 $\mathrm{m}$ deep open pit. Horizontal drains have been installed in the slope. It is evident from these photographs that the hydrogeological characteristics of these two materials would be widely different, with:

- Rock mass - hydraulic conductivity ranging from very low (intact rock) to moderate to high (in continuous joint) and with very low storage.

- Structure - low to moderate hydraulic conductivity and medium to high storage. 


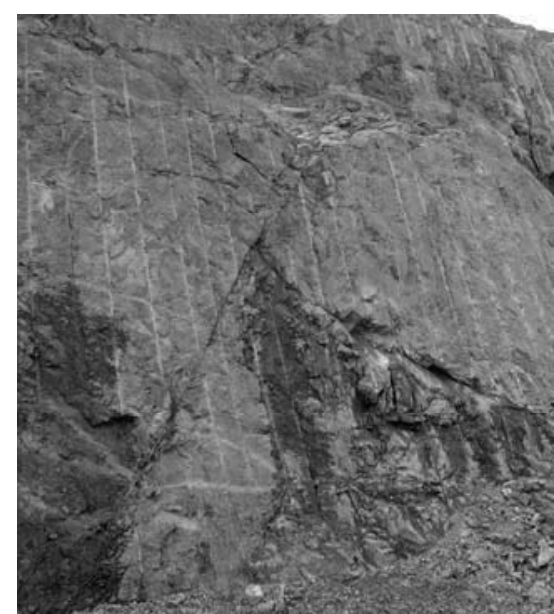

a)

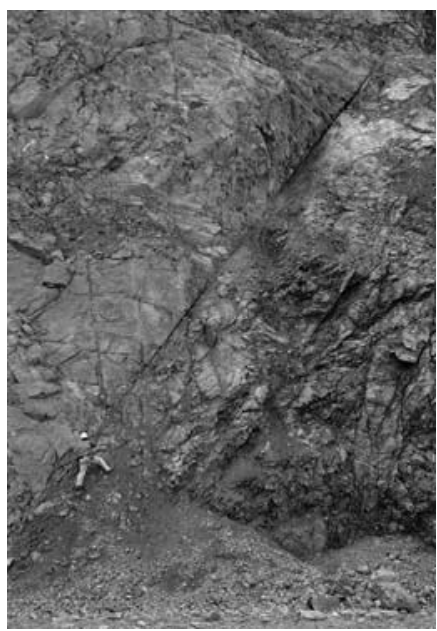

b)

Figure 23 Hydrogeological character of rock mass and structure; a) Broadly jointed high strength rock mass and b) major thrust fault with brecciation of rock mass. Refer to Figure 24 for depressurisation

Figure 24 illustrates the piezometric responses over time and the general degree of depressurisation that has been achieved in these rocks.

(a)

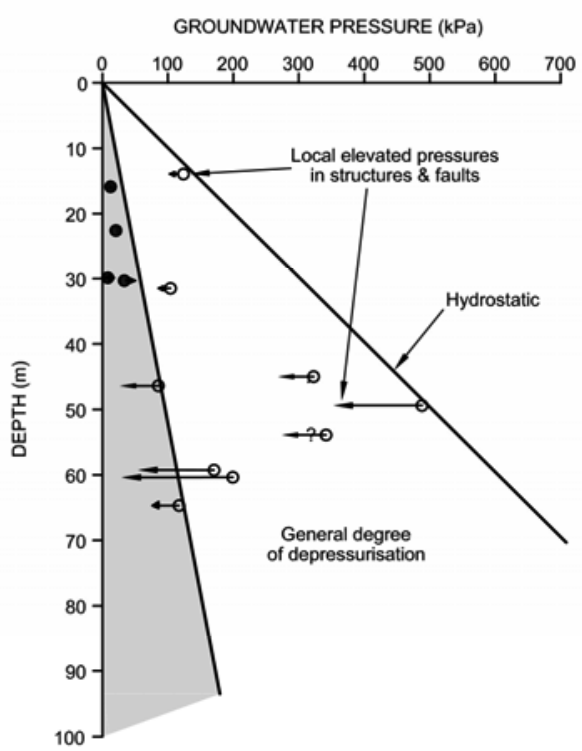

(b)

STRUCTURE

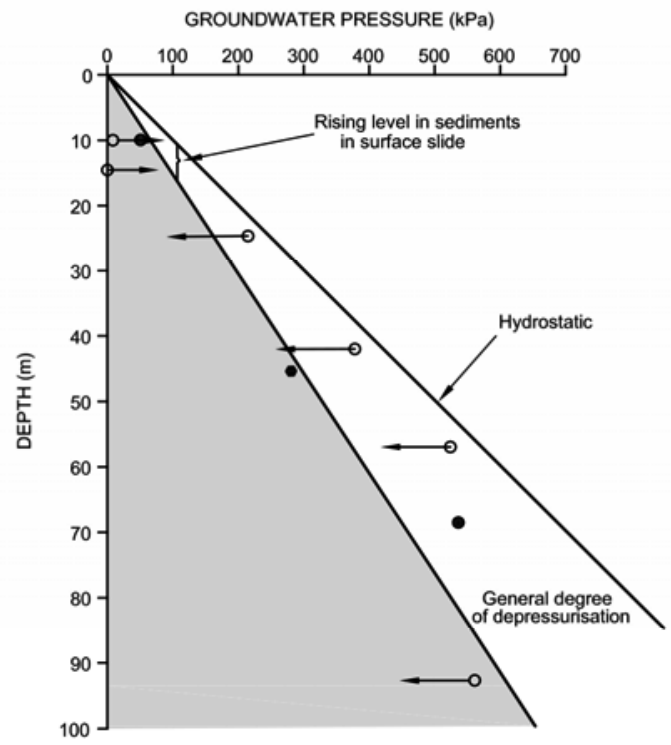

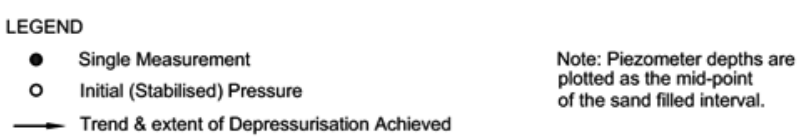

$\longrightarrow$ Trend \& extent of Depressurisation Achieved

Figure 24 Hydrogeological response of general rock mass and structure to natural drainage and horizontal drains. These rock masses are illustrated in Figure 23 
It is often reported that faults are conduits for flow parallel to the structure and barriers to flow in a direction normal to the structure. This example would indicate this experience does not apply universally. This data would also support the concept that depressurisation may be effectively conceptualised in terms of the Coefficient of Consolidation, Figure 22.

The importance of this example for analysing the stability of slopes is that structures may have high pressures, within a generally depressurised rock mass.

\subsection{Rainfall response}

\subsubsection{Natural slopes}

Experience with natural landslides has shown it is very difficult to establish the correlation between rainfall and triggering of slides. Various studies have shown, Fell et al. (2000) that:

- Initially in Hong Kong, there was no correlation between antecedent rainfall and landslides.

- But subsequently for Hong Kong correlations using multiple rainfall gauges did give a correlation.

- There was a delay of several days reported between rainfall and landsliding in Salvador, Brazil.

- However subsequent to these studies it has now been observed in a number of locations that landslide activity results from the combined effects of antecedent rainfall and rainfall intensity.

\subsubsection{Rock mass character, rainfall response and hydromechanical coupling}

Figure 25 shows the general character of two very different rock masses that were excavated in the upper levels a $90 \mathrm{~m}$ high pit slope at an overall angle of $30^{\circ}$. The rock masses comprised a closely jointed bedded sediment a) and a broadly jointed high strength Monzonite, with very widely spaced continuous structures b). On the pit wall these comprise the eastern and western rock masses respectively.

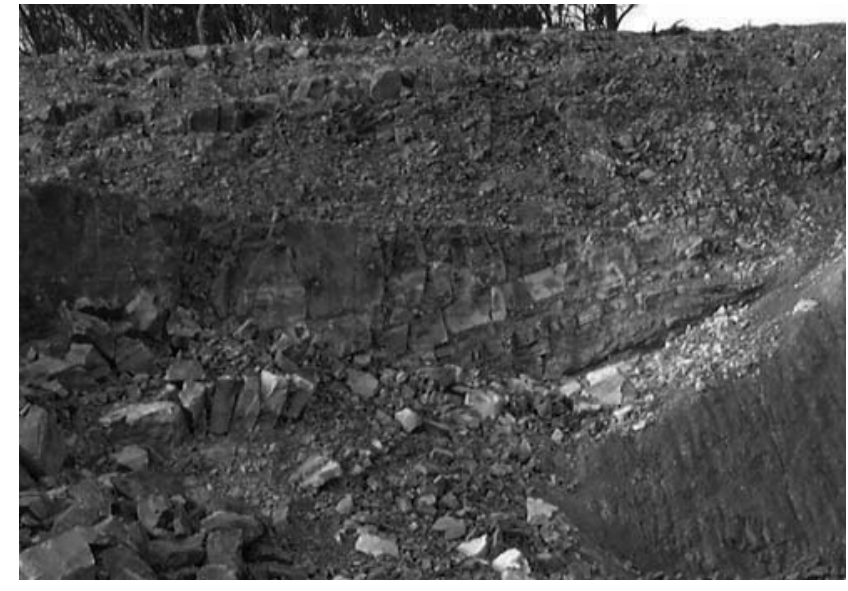

a) East Failure

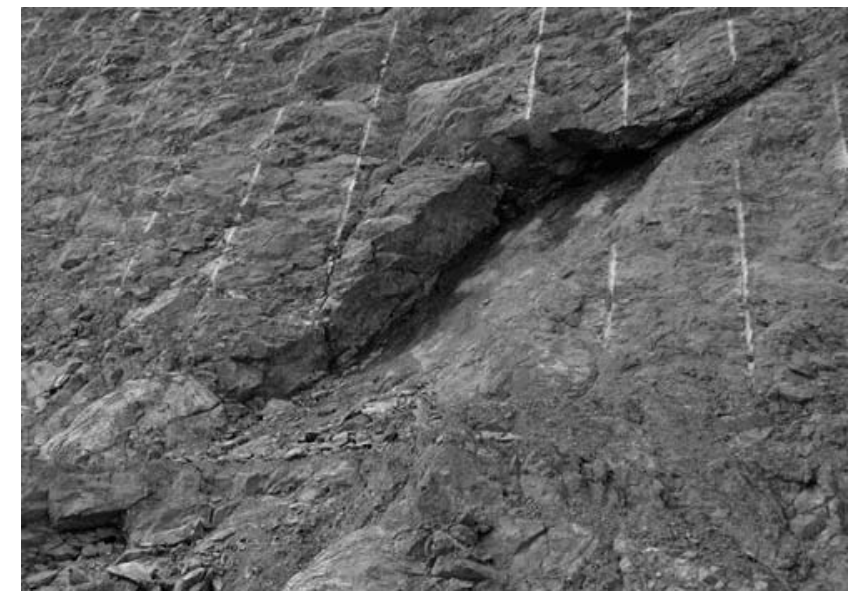

b) West Failure

\section{Figure 25 Examples of two vastly different rock masses involved in failures with particular rainfall responses}

The rock mass defects were generally steep with the exception of a set of faults and shears with a dip direction $35^{\circ}$ away from the dip direction of the pit wall, Figure 26. This is well outside the limit for which plane sliding would normally be considered an issue. 


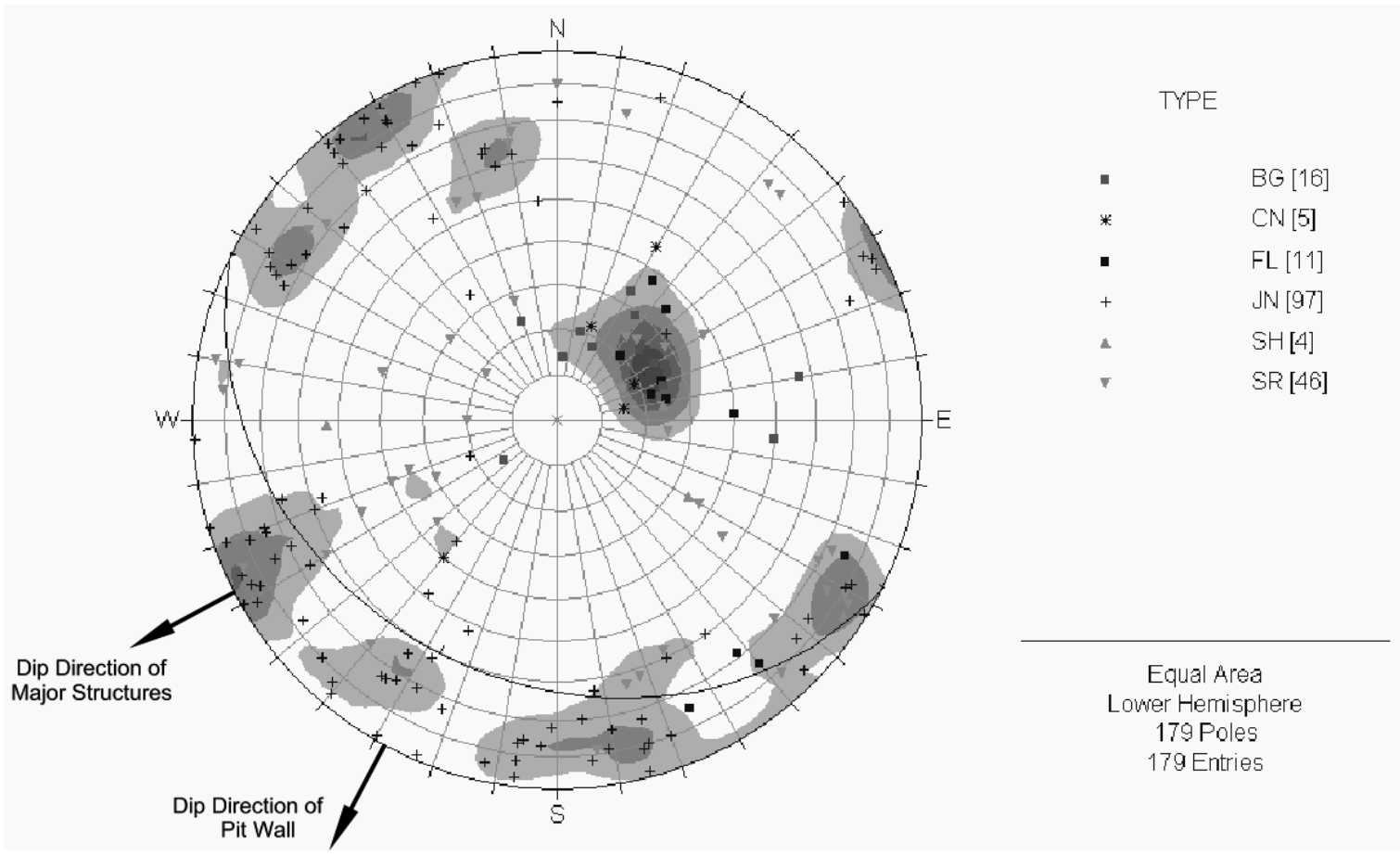

Figure 26 Structural data for failures in rock masses shown in Figure 25

Figure 27(a) shows the correlation of rainfall with pit wall failures in each rock mass. The failures were larger in the eastern rock mass (jointed sediments) up to 220,000 t, and smaller in the Monzonite; up to 65,000 tonnes. This correlation shows an extreme sensitivity even for quite small rainfall events.

Figure 27(b) shows the piezometric response for both the eastern and western rock masses. The jointed sediments show piezometric responses coincident with the rainfall and rises of about $16 \mathrm{~m}$, together with a small longer term semi-permanent rise. This is also in agreement with observations at the time that showed no runoff during rainfall. By contrast in the widely jointed Monzonite there was no piezometric response despite the fact that failures were occurring on structures. This highlights the general observations in Section 7.3 regarding the prediction and modelling of depressurisation in medium scale slopes.

This is an example of Type 4 Hydromechanical coupling.

\subsubsection{Effects of catchments on pit slopes and hydromechanical coupling}

One question often facing open pit designers is what effect catchments have on rainfall runoff response and failures. The rock mass shown in Figure 25(a) was intersected by a haul road at 10\% grade, which was left in the slope for future possible access. As noted earlier, the rock mass was broadly jointed, but the structures were continuous and with a small aperture. One such structure cut across the haul road and a medium scale failure occurred during a small rainfall event (about $20 \mathrm{~mm}$ ) even though the structure was oriented unfavourably for sliding to occur. The intersection of the structure and the haul road was about two thirds of the way along the haul road so even though the rainfall was small there was a significant catchment and potential to channel a significant proportion of the runoff into the structure.

Catchments have the potential to concentrate runoff resulting in indirect Hydromechanical Coupling, Type 4. 


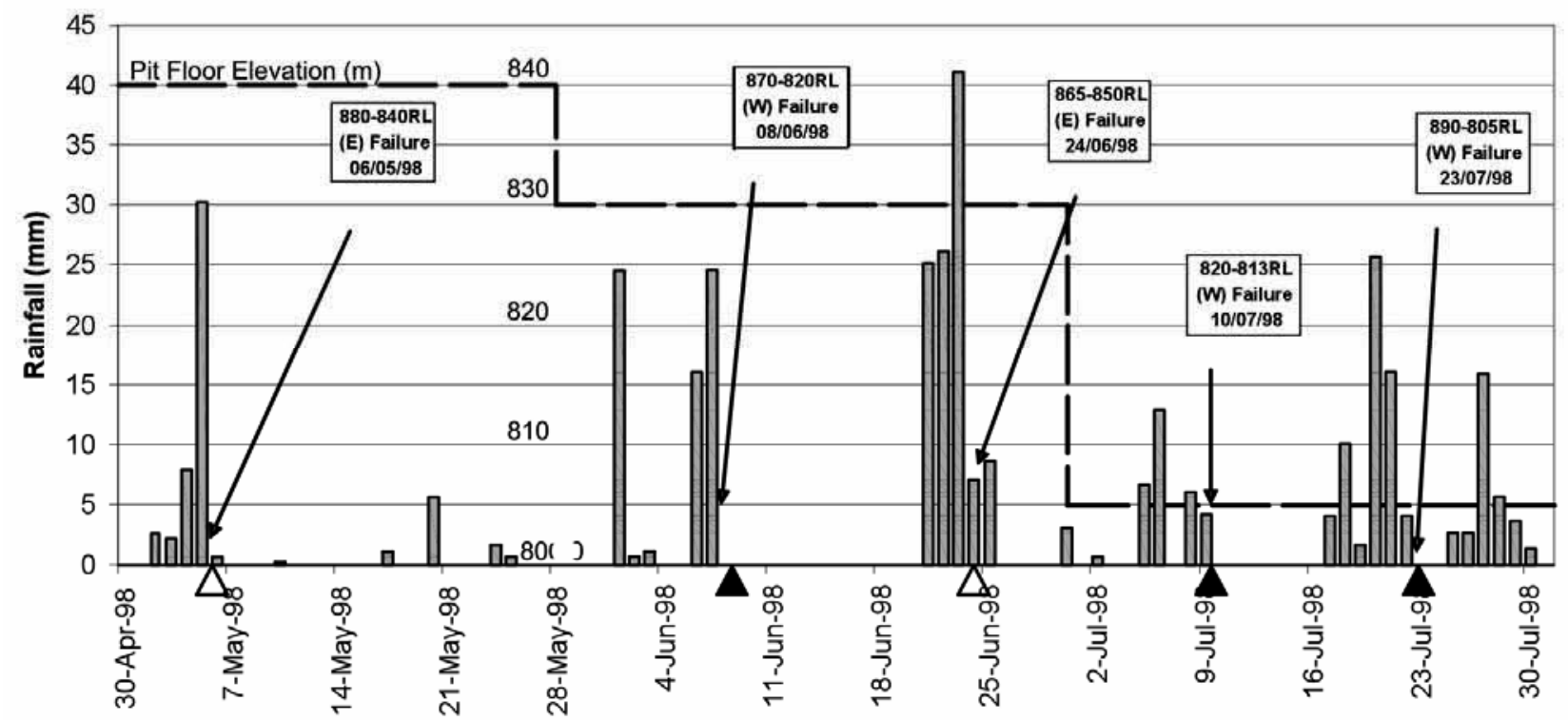

$\triangle$ Eastern Failure

Western Failure

Date

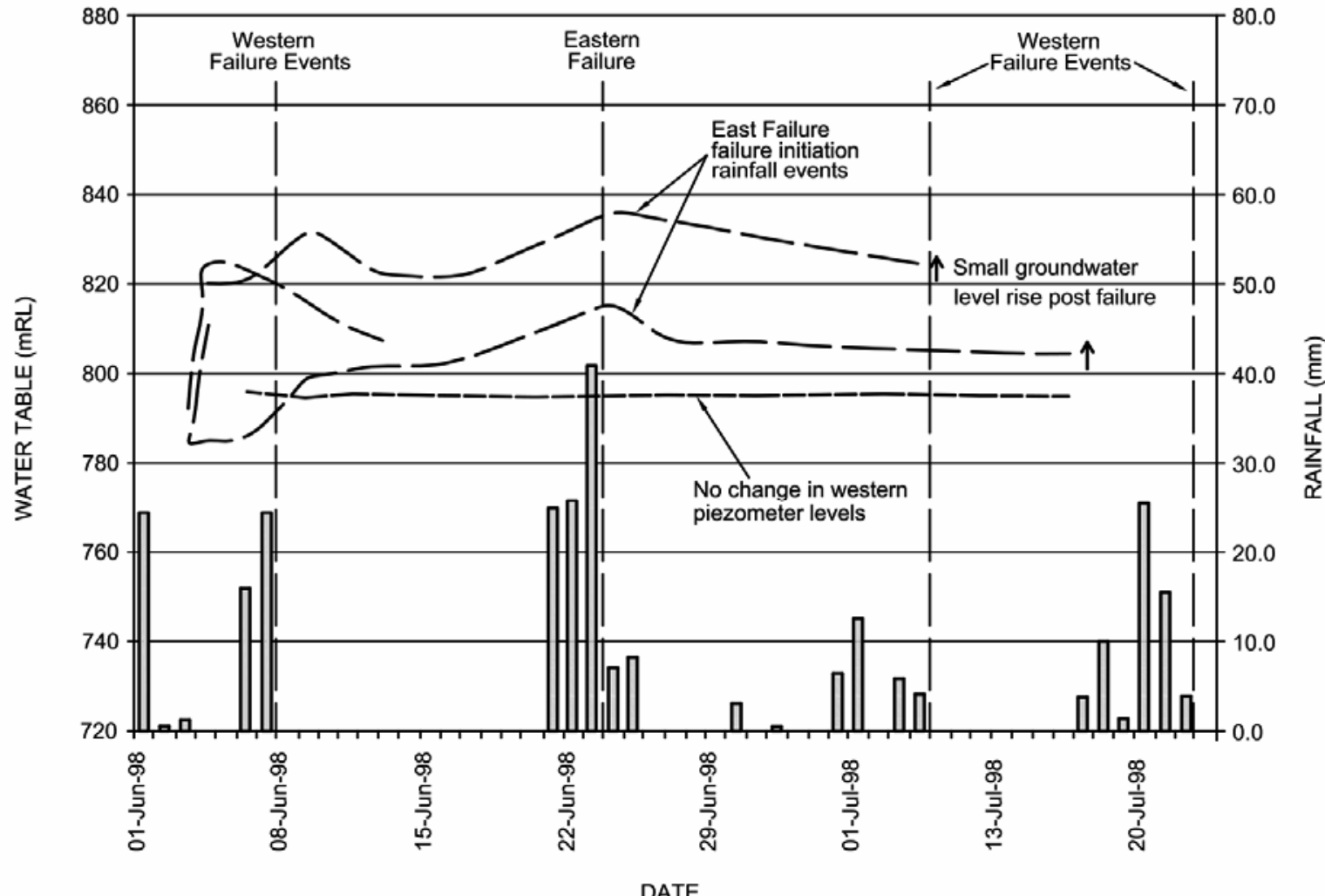

LEGEND

- - West Failure Piezometers

— East Failure Piezometers

Figure 27 Relationship between rainfall and failures a) and failures and groundwater responses to rainfall b) 


\subsubsection{Rainfall, pit slope movements and hydromechanical coupling}

Figure 28a) shows a section through a $200 \mathrm{~m}$ high slope excavated at an overall angle of $35^{\circ}$. The geology comprises bedded shales and Dolerites dipping towards the south out of the slope. Mapping shows a large number of joints, beds and faults, Figure 28(b) dipping to the south at angles generally equal to or steeper than the overall slope. The existing groundwater level is quite deep, 65 to $100 \mathrm{~m}$.
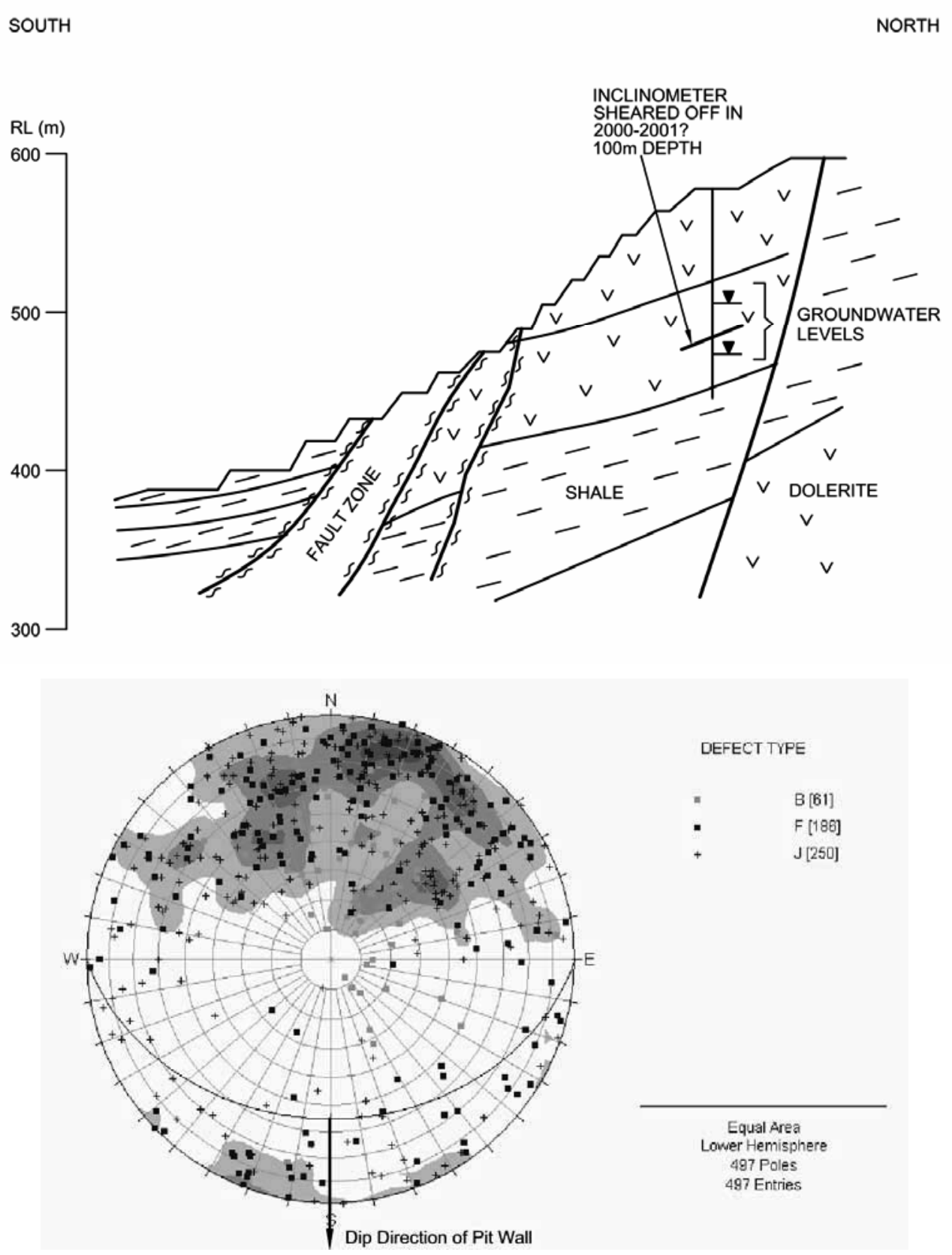

Figure 28 Section through the slope showing groundwater levels and general depth of slope movements a) and structural data $b$ )

The site is in the northwest of Australia and subject to cyclonic rainfall patterns in the summer months.

Figure 29a shows the rainfall and piezometer levels recorded over a period of 6 to 7 years. Figure 29(b) is the data for one survey prism located at about $530 \mathrm{mRL}$ (Figure 28(a)) in the upper middle of the slope. This data shows that during 1999 there was a very small amount of creep, about $0.03 \mathrm{~mm} /$ day. 

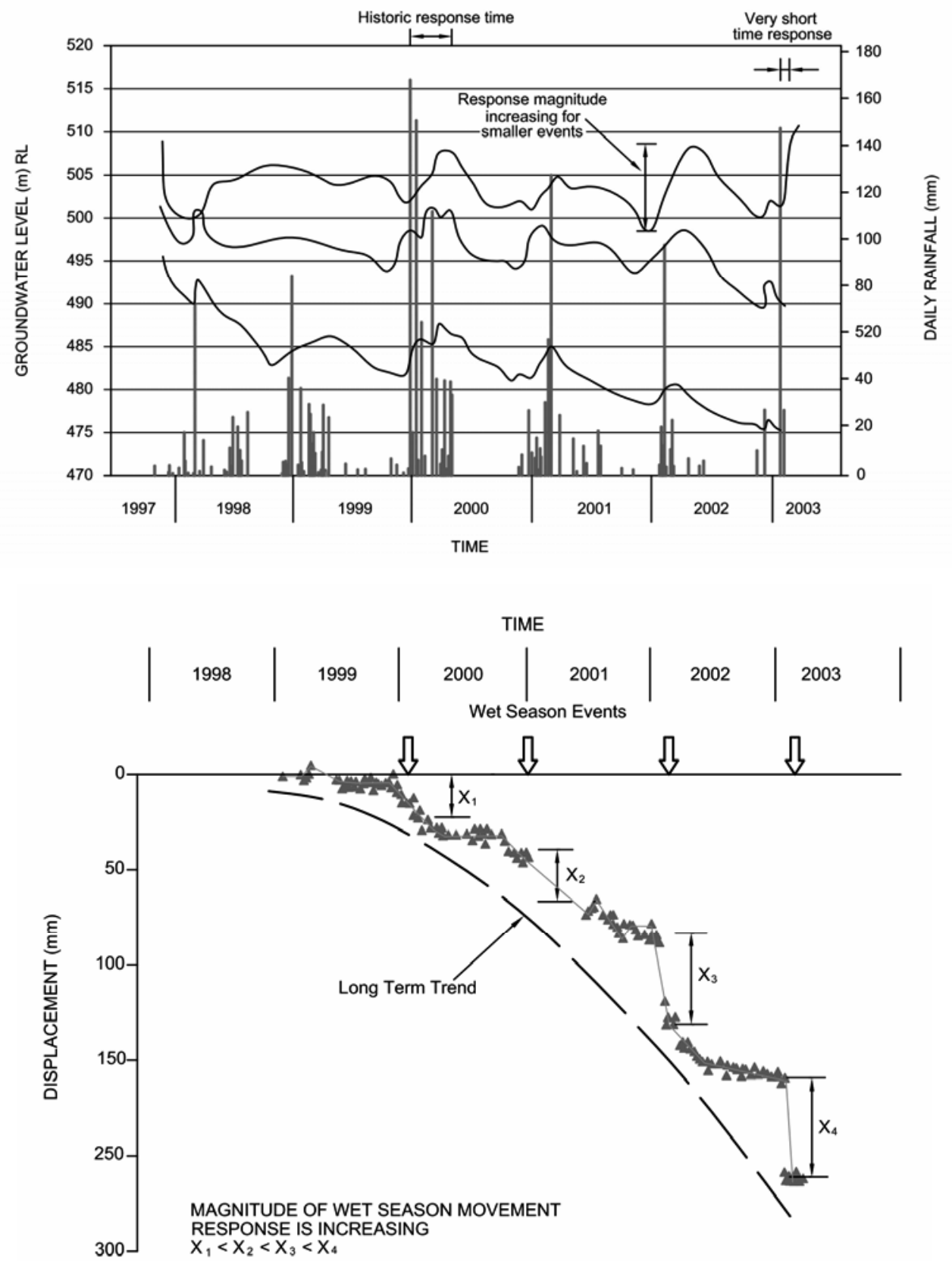

Figure 29 Relationships between rainfall and groundwater responses over time; a) and rock mass movements b)

The two intense rainfalls in early 2000 resulted in a $35 \mathrm{~mm}$ global movement of the slope and sheared off an inclinometer at $100 \mathrm{~m}$ depth (Figure 28a). This was Direct and Indirect Hydromechanical coupling Types 2 and 3. The initial movements caused an increase in the aperture and hydraulic conductivity of the rock structures. Thereafter smaller seasonal rainfalls resulted in:

- Progressively larger movements for smaller rainfall events, Figure 29(b).

- Increasing magnitude of groundwater level rises for smaller rainfall events, Figure 29(a).

- Reduced response times for groundwater level rises as a result of rainfall events, Figure 29(a).

Comparison of the timing of the movements and the timing for rises in groundwater levels shows that transient groundwater pressures in the rock mass above the deeper groundwater table must have played a major role in the global movements. 


\subsubsection{Statistical correlation of rainfall and pit slope failures}

Figure 30 shows the failure size distribution data for a very large open pit in Asia. The rock mass is of variable strength, medium to high, and closely jointed. The rock mass is also intersected by many thin continuous faults and shears. The mine has pronounced wet and dry seasons during the year. Mining of consecutive expanding pit shells over a number of years resulted in many failures, Figure 30 . The failures were up to $200 \mathrm{~m}$ high but generally less than $100 \mathrm{~m}$.

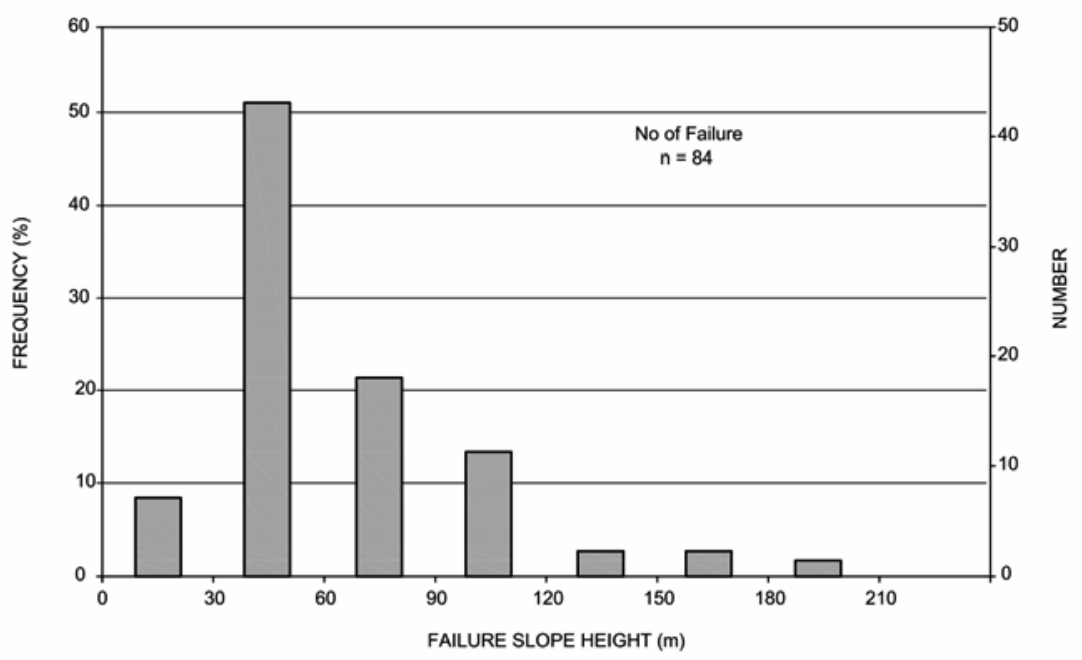

Figure 30 Distribution of slope heights involved in failures

Figure 31 shows the correlation between failure occurrence, the 7 day antecedent rainfall and rainfall on the day of the failure itself for a population of 137 failures. This correlation shows:

- $85 \%$ of failures were associated with rainfall.

- $60 \%$ of the failures were associated with antecedent rainfall in the seven days preceding the failure.

- $25 \%$ of the failures were associated rainfall on the day of the failure only.

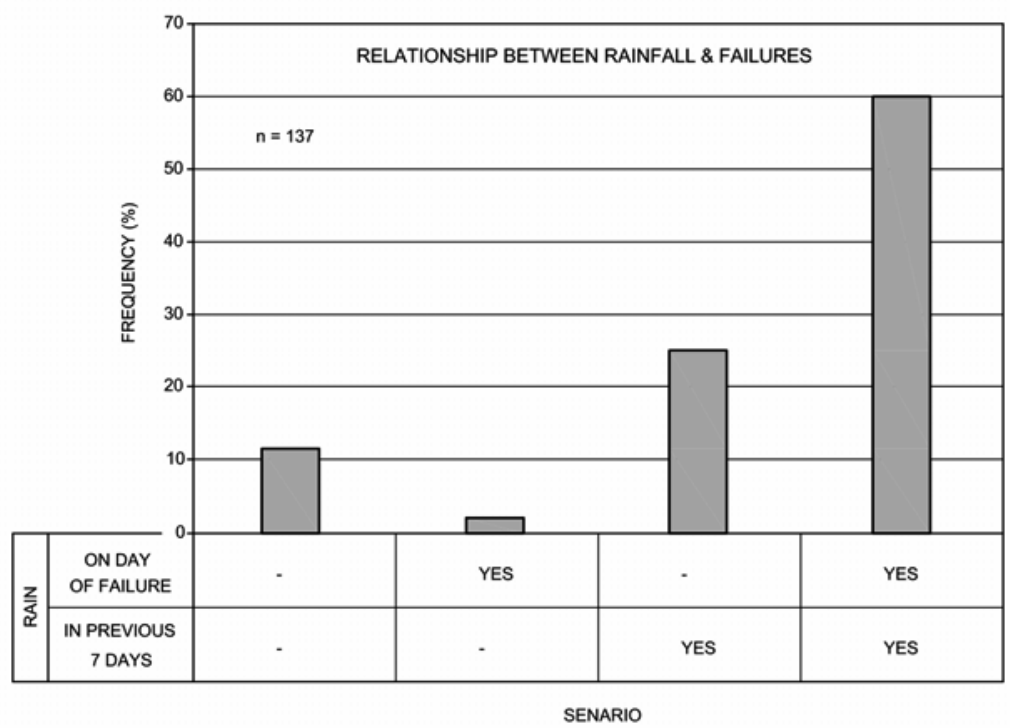

Figure 31 Statistical evaluation of failure data for rainfall on the day of the failure and in the preceding seven days 
Figure 32 presents this same data but expressed as the likelihood of a certain rainfall event occurring and the likelihood of a failure being associated with that rainfall event.
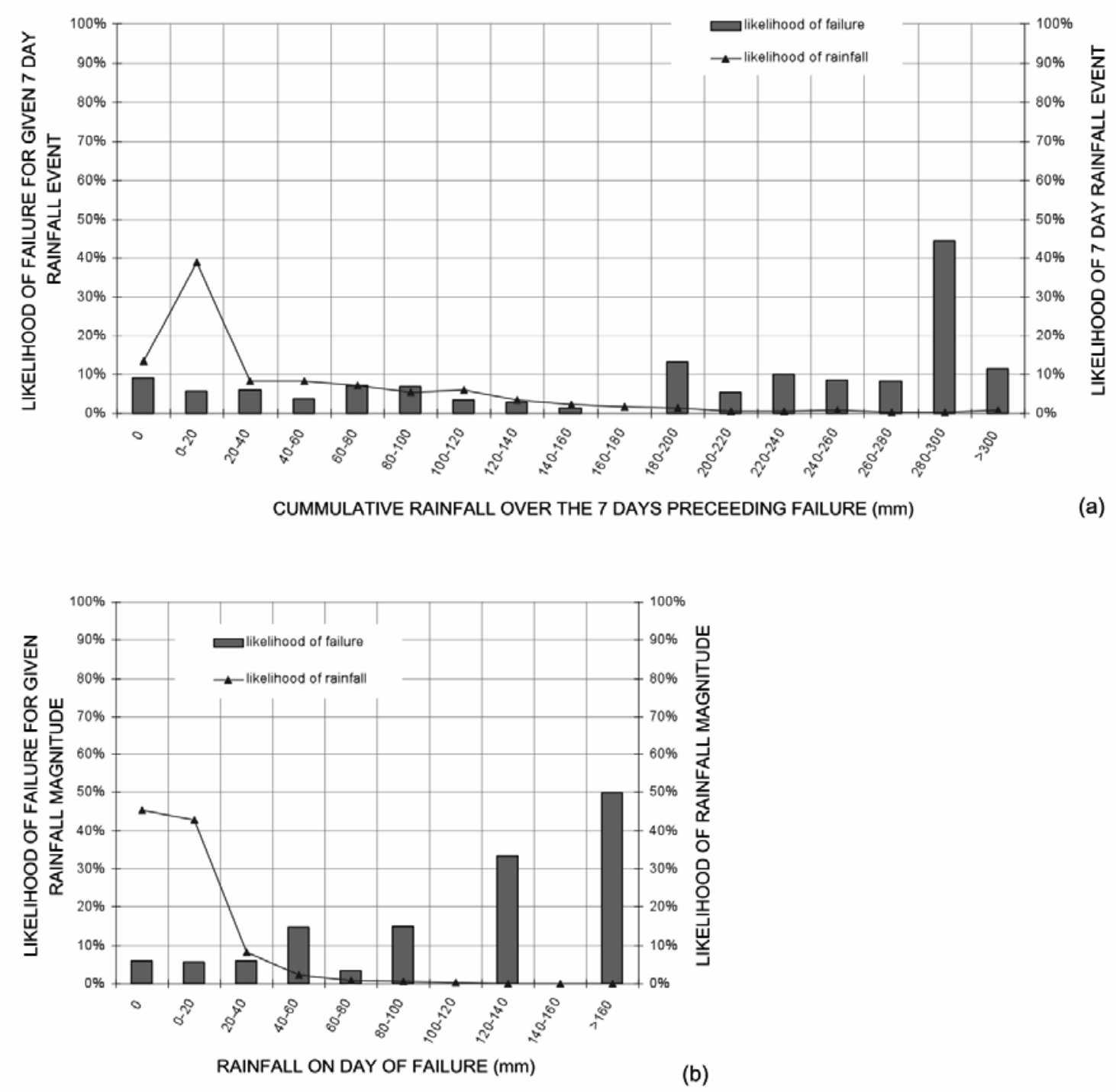

Figure 32 Probability of a failure occurring due to either a 7 day cumulative rainfall magnitude 32(a); or a single day rainfall 32(b)

In terms of antecedent rainfall in the 7 day period prior to failure the data shows, Figure 32(a):

- Although $52 \%$ of the 7 day rainfalls are less than $20 \mathrm{~mm}$, the likelihood of a failure occurring is 5 to $10 \%$. These failures were probably going to happen in any event even without rainfall.

- There is an increased likelihood of failure with the larger 7 day rainfall events $(>180 \mathrm{~mm})$ generally $10 \%$ increasing locally to $45 \%$.

The correlation for rainfall on the day of the failure shows, Figure 32(b):

- $87 \%$ of days have either no rainfall or rainfall less than $20 \mathrm{~mm}$. The chance of failure on any one of these days is about 1 in 20 .

- Although daily rainfalls greater than $80 \mathrm{~mm}$ are less than $3 \%$ of the total days, the chance of a failure occurring on one of those days is high, 15 to $50 \%$. 
The inferences from these correlations are:

- The 7 day antecedent rainfall correlation indicates that transient groundwater pressures in the zone above the groundwater table are playing a role in stability at this medium scale.

- The failures for high daily rainfalls are probably associated with transient groundwater pressures in structures due to local rainfall runoff.

- The critical rainfall event in a seven day antecedent rainfall followed by a daily rainfall in excess of about $80 \mathrm{~mm}$.

\subsection{Hydromechanical coupling and stored water}

Figures 33 to 35 show the slope geometry and rock mass character for a $120 \mathrm{~m}$ high slope. The slope initially developed two lines of arcuate cracks and small scarps after a period of creep movement. The mine undertook blasting to unload the slope, Figure 35. The area to be blasted was intersected by a major structure, Figure 34, that coincided with one line of cracks, formed the rear of the eventual failed mass, and passed through the blast, Figure 33. Straight after the blast, the pond in the foreground of Figure 34 drained and the slope failed. This is an example of Indirect Hydromechanical Coupling, Type 4.

\section{SECTION 10190N}

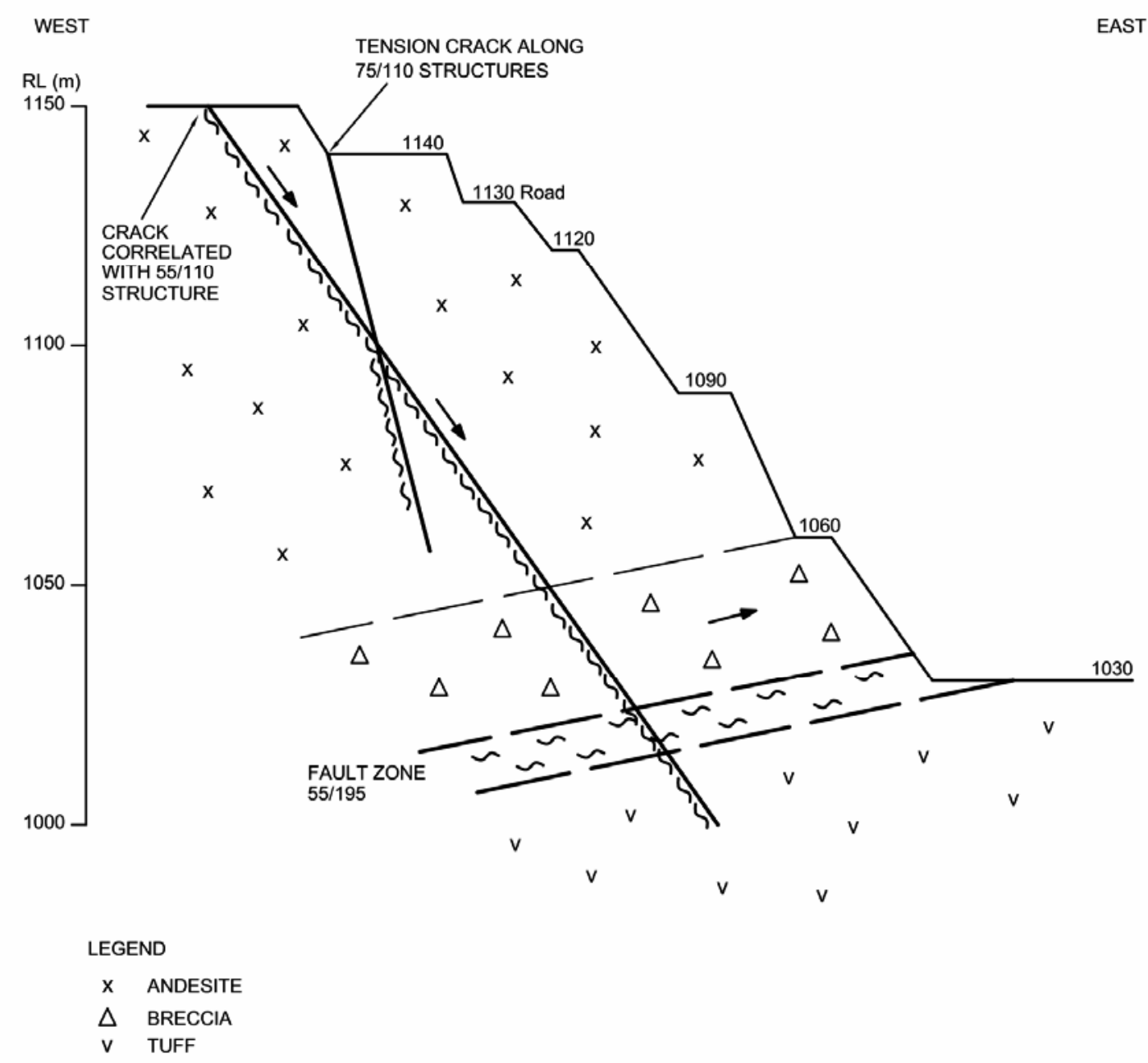

Figure 33 General geology of the slope 


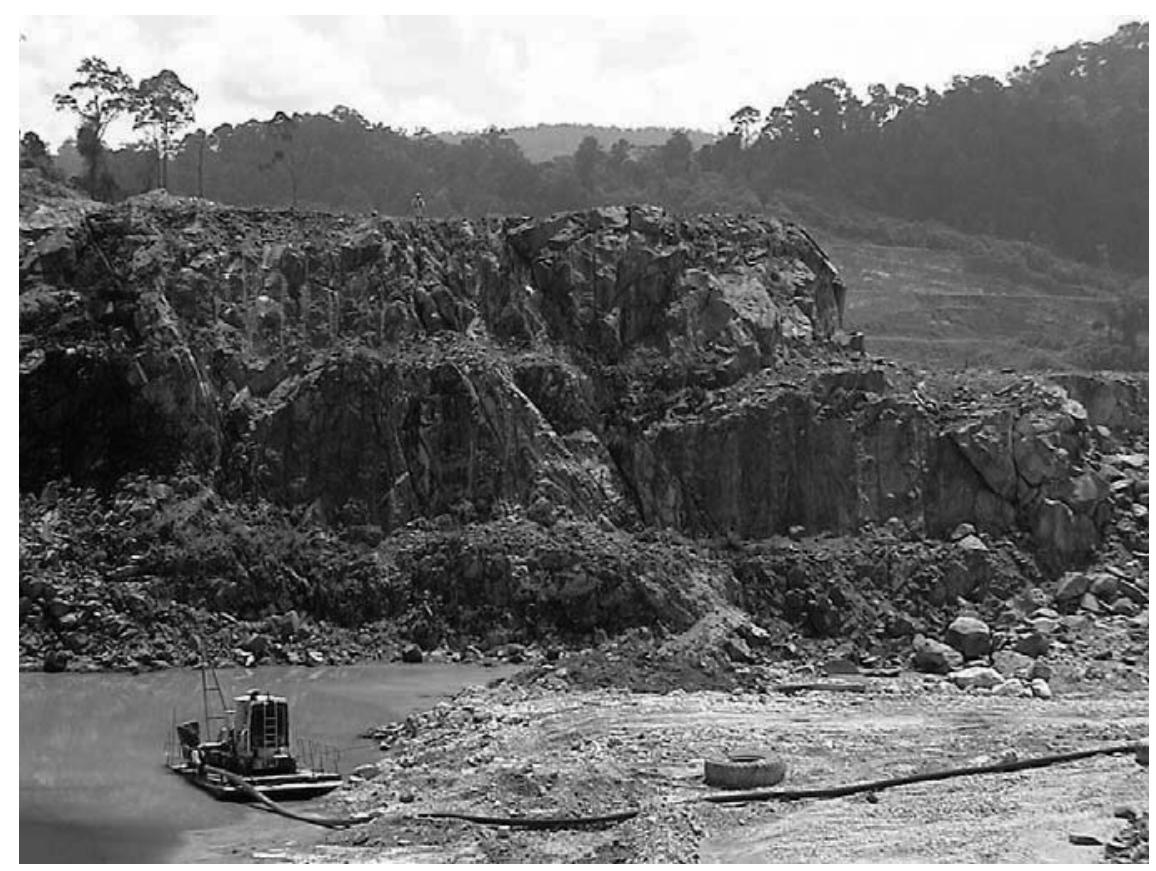

Figure 34 Relationship between slope and ponding - note blasting took place on bench where the observer is standing

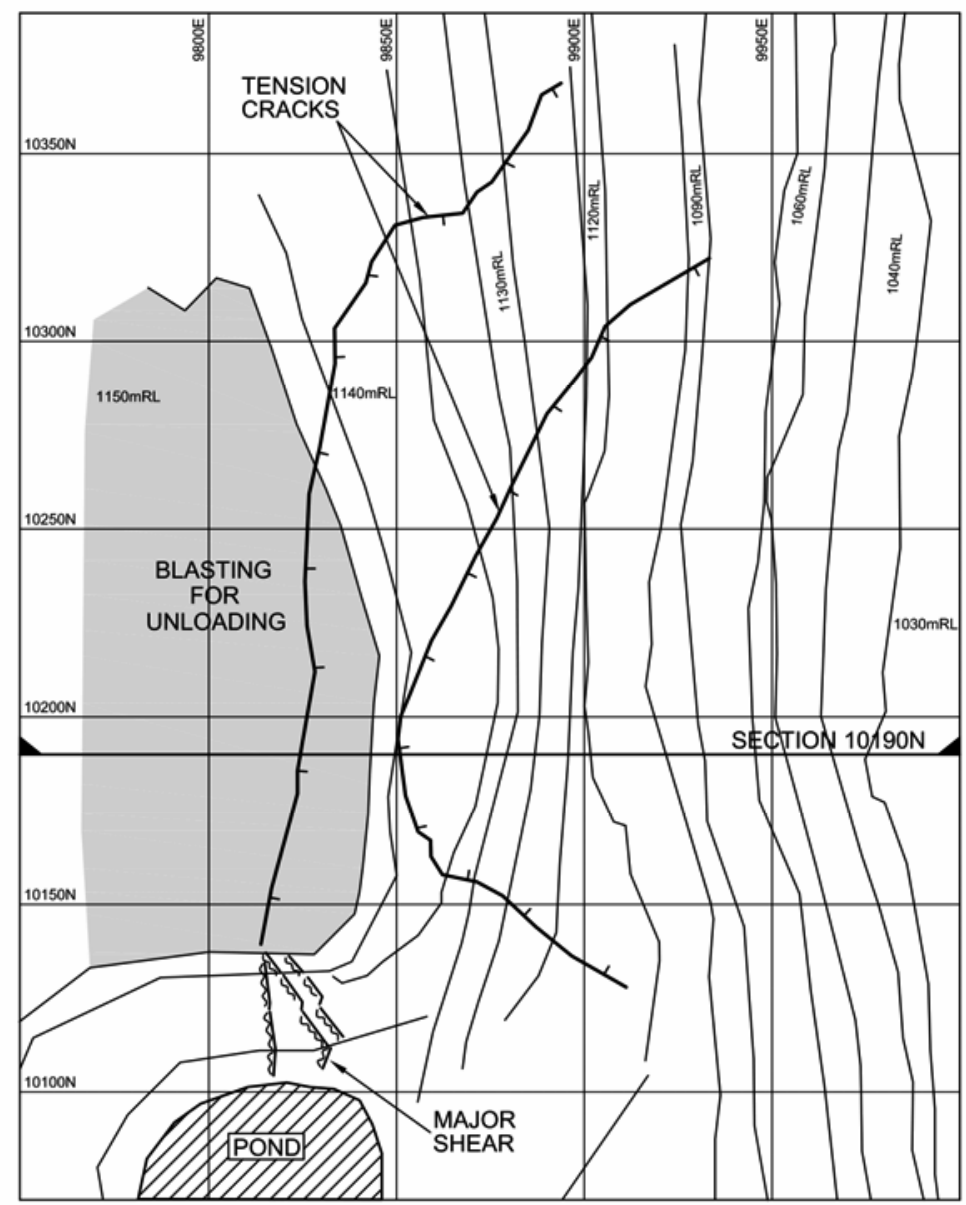

Figure 35 Plan of failure showing section location, location of blasting, tension cracks and pond 


\subsection{Unloading}

By definition a rock mass contains structures, which are deformable and often fluid filled. Deformation of this rock mass can occur either as a result of a change in the external loading or a change in the pore fluid pressure. Under external loading the rock mass will compress resulting in a smaller overall rock mass volume and hence compression of the pores or the structures and the fluid contained therein, Figures 4 and 5. If this loading is rapid the volume reduction is carried by the pore fluids resulting in increased pore fluid pressures largely because there is insufficient time for the fluid to escape. This is undrained loading. However if the loading is slow the fluid has time to escape and excess pore pressures are not generated. This is drained loading. These are an example of Direct Hydromechanical Coupling, Type 1.

Unloading is the opposite of loading but the mechanics are the same. The unloading causes an instantaneous decrease in the stress and an increase in the rock mass volume, which expands because of the stress reduction. The pores or fractures in the mass expand and the water in the pores of the saturated rock mass is drawn into tension because water is incompressible. Because the unloading is rapid there is insufficient time for this tension, which is manifested as a negative pore pressure, to be satisfied and hence the pore water pressure is reduced. Over time water flows in from adjacent areas to satisfy this negative pore pressure. This phenomena has mainly been observed in civil engineering, where it is well established. In materials with a low to very low hydraulic conductivity the equilibration time has been of the order 50 to 100 years.

Figure 36 shows this effect for an excavated slope, Bishop and Bjerrium (1960) and Lamb and Whitman (1969).
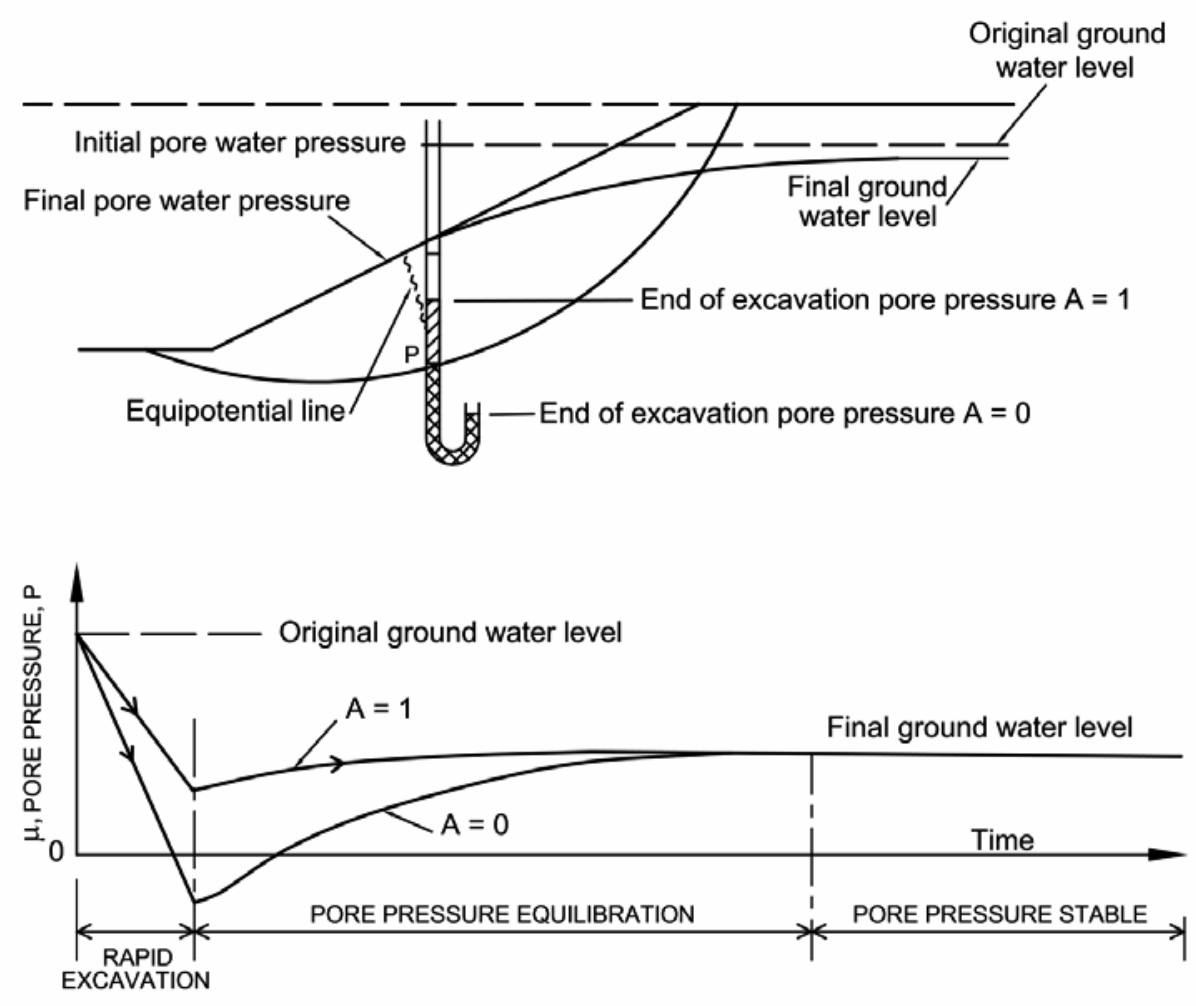

Figure 36 Unloading and pore pressure equilibriation after excavation (after Bishop and Bjerrum, 1960)

The author has measured reduced pore water pressures in a coal mine footwall excavated some 80 metres below the groundwater table. The rock type was a shale. These pressures indicated a level some $20 \mathrm{~m}$ below the pit floor and were measured about 15 years after mining was completed in that area. The author has also measured pore pressure reductions due to unloading in a trial coal pit, Burman and Sullivan (1985) and O’Brien and Sullivan (1988). 
Over time the water demand created by this unloading will dissipate due to transient groundwater inflows from surrounding areas.

However it should be understood when assessing unloading effects, that factors such as the in situ stress and the three dimensional pit geometry mean that the degree of depressurisation due to unloading is related not to the change in vertical stress but to the bulk stress change at the particular point around the excavation.

Many open pits undergo a semi-continuous process of unloading due to multiple cutback development. Based on the theory of Direct Hydromechanical Coupling Type 1, when a cutback bench is excavated there is a significant reduction in stress. The stress reduction even from a single $15 \mathrm{~m}$ high bench may be very large, around $400 \mathrm{kPa}$.

\subsection{Liquefaction or undrained loading}

There have been some recent relatively large pit wall failures that have exhibited what has been interpreted as liquefaction behaviour post collapse or failure, with very large and catastrophic post failure deformations.

Experience has shown that in order for liquefaction to occur the rock mass will have to show at least two factors:

- Firstly become disaggregated to a relatively fine grained granular, non plastic, particle size.

- Secondly have had a very significant increase in the overall percentage of water contained in the failing mass.

One of the large uncertainties at this stage is related to the fact these failures must have started out as a reasonably sound rock mass, certainly better than a finely disaggregated particulate. Presumably the rock mass condition must have been sufficient to have allowed a pit wall to be developed and monitored for some time as the excavation progressed. This means the mechanics of the slope and the mechanisms controlling behaviour must have changed substantially pre and post failure, Figure 17. It is also evident that the type of hydromechanical coupling changed through the process of the changing mechanisms. Liquefaction is an example of Indirect Hydromechanical Coupling Type 4.

There is a broad correlation between degree of runout and degree of fragmentation. Hutchinson (2006) addressed the mechanisms of rock fragmentation and related it in part to explosive fracturing of the individual rock particles under loading. However, these processes are normally associated with very large scale rock avalanches.

As noted above one of the two fundamental factors is to be able to develop a significant degree of saturation and one that is commensurate with the particle size and the particle size distribution of the failing mass. This significantly increased water content does not necessarily have to be developed in the whole of the mass, but at least in the lower part of the slope where any dynamic loading caused by sudden partial or complete collapse can lead at least in part to sudden development of high pore pressures due to Indirect Hydromechanical Coupling Type 4. The water content also has to be high enough to generate elevated pore pressures in the failing mass. If significantly less than saturated the mass is unlikely to be able to generate the elevated pore pressures required for liquefaction.

Figure 19 is a plot of velocity versus time for a hypothetical pit slope and illustrates what happens over time as the slope passes through the various slope movement stages. At any time from the stage of elastic movement through to post collapse, the velocity of many pit wall failures decreases to zero and the slope or failure would be regarded as stable. However there is a class of failures that continue post collapse to move at high velocities and it is these failures that have been classed generally as liquefaction or undrained loading type failures.

Figure 19 also illustrates the potential changes in hydraulic conductivity, water content, infiltration capacity and response to rainfall that occurs as the slope passes through these movement stages. Changes in these parameters can lead to an increased potential for large post failure deformations after collapse.

This process operates to a lesser or greater degree in all pit slope failures where movement occurs over a period of time and encompass some rainfall interaction. The two case studies described in Sections 8.3.2 and 8.3.3 both contain elements of these factors. 
Assuming the failure is in a rock, then the water content also has to be available to contribute to the mechanism, in that it is unlikely to contribute to liquefaction if contained wholly or in large part in the micropores of the rock substance. The water probably has to be available either in rock structure or in the spaces between the aggregate. However, Hutchinson (2006) has reported flow slides in "soft" high porosity $(40 \%)$ rocks (chalk) that are fully or nearly saturated. The mechanism attributed to this flow sliding is impact collapse.

The Thredbo Landslide which was a liquefaction type failure in a clean, fine to medium grained sand had a moisture content around $30 \%$. Conversely data from one of the recent pit slope liquefaction failures indicated a moisture content in range from 12 to $18 \%$, pers. comm. Assuming a high initial water content of around $1 \%$ for the rock mass before failure, then it is clear that a very substantial increase in water content is required for liquefaction. This water needs to be retained in the failing mass and increase over time despite slope movement continuing to occur and without the slope reaching the drainage threshold.

It is clear that the mechanisms controlling liquefaction type failures of a rock mass are as yet only poorly understood and there are many uncertainties.

\section{Conclusions}

This paper presents a review of the general state of the art in the understanding of the interaction between water and pit slopes. Pore water controls the deformation of porous media and in turn the deformation of porous media affects pore fluid pressures and flows, mainly by altering hydraulic conductivity and storage. This is a two way process and is a fundamental aspect of the dynamic interaction that affects the deformation of pit slopes and the groundwaters contained within them, including rainfall runoff. This process is termed "hydromechanical" coupling.

Many pit slopes undergo large unrecoverable deformations, which are a complex function of the three dimensional stress state, time, geotechnical properties of the rock mass, the structural geology, groundwater pressures and excavation practices. The deformations of pit slopes, which are largely inelastic with creep and slip on structures, cause irreversible changes in the rock mass and hydraulic properties of the mass and are largely indirect hydromechanical coupling. Most of the geotechnical processes of pit slope deformation and behaviour can only be fully understood by considering the coupling between groundwater and deformation and this is still not widely appreciated. Because the hydraulic conductivity of rock defects is proportional to the third power of the aperture, small changes in stress will result in quite significant changes to the hydraulic conductivity of most structures. Open pit slopes need to be differentiated from natural slopes because the physical properties are changing over the life of the slope and the newly excavated slopes are subject to new environmental influences from water effects, such as rainfall runoff.

Experience has shown there are five possible stages of pit slope movements:

1. Elastic.

2. Creep.

3. Cracking and dislocation.

4. Collapse (failure).

5. Post failure deformation.

The initial stages of movement (Stage 1 and 2) have great significance for the hydrology of the rock mass, because movement changes the water pressure and the hydraulic parameters: hydraulic conductivity and storage. The emphasis for slope designers has traditionally been on the prediction and timing of failure. However, experience has shown that adequate recognition of the early stages of movement are very important for the effective management of the slope. In addition the recent experiences with some tragic pit wall failures has reinforced the need to place equal emphasis on predicting the post collapse performance.

Experience has shown that the existing classification systems used for assessing pit slope movements do not adequately capture all the patterns and types of movement behaviour. In order to better explain the full range of movements experienced by pit slopes and to capture the potential for catastrophic movements post failure it is necessary to sub-divide pit slope movements into three periods; pre-failure movements, failure 
movements and post-failure movements. This system has three benefits, it allows a more complete description of the wide range of movement types that occur leading up to the onset of failure; it allows the wide range in failure acceleration periods to be indicated and it also allows the range of post failure deformations behaviours to be illustrated, including the velocity.

Threshold velocities are presented for planning and management of moving slopes and failures.

Mining reduces the lateral and vertical stress resulting in strains that generally increase porosity, aperture and connectivity between fractures. Over the mine life other deformations also occur which further alter the stress state and fluid pressures leading to transient flows. Many pit slopes are now approaching and exceeding $1000 \mathrm{~m}$ depth, and the roles of transient and partial pore pressures in the upper levels are becoming increasingly important.

In most large deep open pit slopes we are generally dealing with four possible groundwater design conditions:

- Unsaturated conditions with no pore pressures above the water table.

- Transient saturated conditions in the upper levels with partial pore pressures due to transient rainfall infiltration response.

- Saturated conditions below the groundwater table with full hydrostatic pore pressures.

- Saturated conditions below the groundwater table with partial pore pressures, less than hydrostatic, usually due to an artificial depressurisation or drainage.

In open pits hydromechanical coupling can be direct or indirect, where direct hydromechanical couplings occur through an interaction between the deformation of the slope and the pore-fluid, and indirect hydromechanical couplings occur when these two factors are not directly linked. Wherever there is rainfall and or groundwater, hydromechanical couplings occur to various degrees whenever a mine slope is excavated.

Statistical evaluation of 137 medium scale failures shows:

- The critical rainfall event is a 7 day antecedent rainfall.

- $60 \%$ of the failures are associated with the 7 day antecedent rainfall and with rainfall on the day.

- $25 \%$ of the failures are associated with a 7 day antecedent rainfall only, with no rainfall on the day.

- The 7 day antecedent rainfall correlation indicates that transient groundwater pressures in the zone above the groundwater table are playing a role in stability at this medium scale.

- The failures for high daily rainfalls are probably associated with transient groundwater pressures in structures due to local rainfall runoff.

The large scale performance of pit slopes over the life of the mine is influenced by many environmental factors. Understanding these influences leads to the better recognition of risks and the better management of these risks.

\section{References}

Alonso, E.E., Gens, A. and Delahaye, C.H. (2003) Influence of Rainfall on the Deformation and Stability of a Slope in Overconsolidated Clays : A Case Study. Hydrogeology Journal 11, pp. 174-192.

Biot, M.A. (1941) General theory of three-dimensional consolidation. J. Appl. Phys. 12(2), pp. 155-164.

Bishop, A.W. and Bjerrum, L. (1960) The Relevance of the Triaxial Test to the Solution of Stability Problems. Proc. ASCE Research Conf. on Shear Strength of Cohesive Soils, Boulder, Col., pp. 437-501.

Brown, A. (1982) The Influence and Control of Groundwater in Large Slopes. Proceedings 3rd Int. Symposium Stability in Surface Mining, Chapter 3, pp. 19-39.

Broadbent, C.D. and Zavodni, Z.M. (1982) Influence of Rock Structure on Stability. Stability in Surface Mining, Volume 3, Society of Mining Engineers, Chap. 2.

Burland, J.B., Longworth, T.I. and Moore, J.F.A. (1977) A study of ground movement and progressive failure caused by a deep excavation in Oxford Clay. Geotechnique, Vol. 27 (4), pp. 557-591. 
Burman, B.C. and Sullivan, T.D. (1985) Dewatering and Depressurisation Studies for Development of the Lochiel Open Pit Mine, South Australia. Mine Water, Granada, Spain, pp. 307-324.

Couture, R. and Evans, S.G. (2006) Slow-Moving Disintegrating Rockslides on Mountain Slopes. S.G. Evans et al. (eds.) Landslides from Massive Rock Slope Failure, pp. 377-393.

Fell, R., Hungr, O., Leroueil, S. and Riemer, W. (2000) Keynote Lecture - Geotechnical Engineering of The Stability of Natural Slopes, and Cuts and Fills in Soil. Proc. Conference GeoEng 2000, Melbourne, Australia.

Freeze, R.A. and Cherry, J.A. (1979) Groundwater. Prentice Hall, Englewood Cliffs, NJ 07632.

Glastonbury, J. (1999) Preliminary Study of the pre collapse deformation of cut rock slopes. Unpublished report, School of Civil and Environmental Engineering, University of New South Wales, Sydney (part of Phd study).

Hutchinson, J.N. (2006) Massive Rock Slope Failure: Perspectives and Retrospectives on State-of-the-Ar. S.G. Evans et al. (eds.) Landslides from Massive Rock Slope Failure, pp. 619-662.

Kennedy, B.A. and Niermeyer, K.E. (1970) Slope monitoring systems used in the prediction of major slope failure at the Chuquicamata Mine, Chile. Proceedings Symposium on Planning Open Pit Mines, Johannesburg. A.A. Balkema, Amsterdam.

Lamb, T.W. and Whitman, R.V. (1969) Soil Mechanics. Massachusetts Institute of Technology.

Leroueil, S., Locat, J., Vaunat, J., Picarelli, L. and Faure, R. (1996) Geo Characterisation of Slope Movements. Proceedings 7th International Symposium on Landslides, K. Senneset (editor) Trondheim, Norway, Balkema, Rotterdam. Vol. 1, pp. 53-74.

Louis, C. (1974) Rock Hydraulics. International Centre for Mechanical Sciences. Courses and Lectures - No. 165. Rock Mechanics. Edited by L. Müller.

Martin, D.C. (1993) Time dependent deformation of rock slopes. PhD Thesis, University of London, August 1993.

Neuzil, C.E. (2003) Hydromechanical Coupling in Geologic Processes. Hydrogeology Journal 11, pp. 41-83.

O’Brien, M.D. and Sullivan, T.D. (1988) The Lochiel Trial Pit Design and Excavation. The AusIMM Sydney Branch, Minerals and Exploration at the Crossroads, Sydney, NSW, pp. 125-133.

O’Brien, M.D., Sullivan, T.D. and Redman, P.G. (1988) Design and Operation of the Dewatering System for the Lochiel Trial Pit. The Third International Mine Water Congress, Melbourne, Australia, pp. 721-731.

Pariseau, W.G. (2001) Coupled Geomechanic-Hydrologic Approach to Slope Stability Based on Finite Elements. Slope Stability in Surface Mining, Chapter 11. Published by the Society for Mining, Metallurgy and Exploration Inc.

Rutqvist, J. and Stephansson, O. (2003) The Role of Hydromechanical Coupling in Fractured Rock Engineering. Hydrogeology Journal, 11, pp. 7-40.

Ryan, T.M. and Call, R.D. (1992) Application of rock mass monitoring for stability assessment of pit slope failure. Proceedings of 33rd U.S. Rock Mechanics Symposium, pp. 221-229.

Savely, J.P. (1993) Slope management strategies for successful mining. In Innovative Mine Design for the 21 st Century. Kingston, August 23-26, 1993, pp. 25-34.

Sharp, J.C. (1970) Fluid flow through fractured media. PhD Thesis, University of London.

Small, C.A. and Morgenstern, N.R. (1992) Performance of a highwall in soft rock Highvale Mine, Alberta. Canadian Geotech. Jour., 29, 3 June 1992.

Snow, D.T. (1965) A parallel plate model of fractured permeable media. PhD Thesis, University of California, Berkeley.

Snow, D.T. (1968) Rock fracture spacings, openings, and porosities. J. Soil Mech. Found. Div. ASCE 73-91.

Stacey, T.R., Terbrugge, P.J., Keyter, G.J., and Xianbin, Y. (2003) Extension Strain - A New Concept in Open Pit Slope Stability, and its Use in the Explanation of Two Slope Failures.

Sullivan, T.D. (1993) Understanding Pit Slope Movements. Geotechnical Instrumentation and Monitoring in Open Pit and Underground Mining, T. Szwedzicki (editor). Balkema, Rotterdam, ISBN 9054103213.

Sullivan, T.D. (1994) Mine Slope Design - The Chances of Getting the Answer Right and the Risk of Getting it Wrong. 4th Large Open Pit Mining Conference, Perth.

Sullivan, T.D. (2006) Pit Slope Design and Risk - A View of the Current State of the Art. International Symposium on Stability of Rock Slopes in Open Pit Mining and Civil Engineering. The South African Institute of Mining and Metallurgy. Symposium Series 544. Cape Town, South Africa.

Terzaghi, K. (1923) Die Berechnung der Durchlässigkeitziffer des Tones aus dem Verlauf der hydrodynamischen Spannungserscheinungen. Akad. Wissensch. Wien Sitzungsber. Mathnaturwissensch Klasse IIa 142 (3/4), pp. 125-138.

Wang, H.F. (2000) Theory of Linear Poroelasticity. Princeton University Press, p. 287.

Wyllie, D.C. and Mah, C.W. (2004) Rock Slope Eng. Civil and mining 4th edition.

Zavodni, Z.M. (2001) Time-Dependent Movements of Open-Pit Slopes. SME Proceedings, Denver, Colorado, pp. 8187. 